\title{
1 Fatty acid oxidation impairs macrophage effector functions that control Mycobacterium
}

4 Pallavi Chandra ${ }^{1,2}$, Li He $^{3,4}$, Matthew Zimmerman ${ }^{5}$, Guozhe Yang ${ }^{1,2}$, Stefan Köster ${ }^{6, \#}$, Mireille

$9 \quad{ }^{1}$ Division of Infectious Diseases, Dept. of Medicine; ${ }^{2}$ Dept. of Molecular Microbiology; ${ }^{3}$ Dept.

10 of Pathology and Immunology; ${ }^{4}$ Cardiovascular Division, Dept. of Medicine; Washington

11 University School of Medicine, St. Louis, MO 63110; ${ }^{5}$ Center for Discovery and Innovation,

12 Hackensack Meridian Health, Nutley, NJ 07003; ${ }^{6}$ Division of Infectious Diseases and

13 Immunology, Department of Medicine, New York University Medical Center, New York, NY,

14 10016, USA. ${ }^{7}$ Marc and Ruti Bell Vascular Biology and Disease Program, Leon H. Charney

15 Division of Cardiology, Department of Medicine, New York University Medical Center, New

16 York, NY, 10016, USA.

17

$18{ }^{\#}$ Current affiliation: Sanofi NA BT Lab, 270 Albany St, Cambridge, Massachusetts, 02139

19 Current affiliation: Department of Biochemistry, Microbiology, and Immunology, University of

20 Ottawa, Ottawa, Ontario, Canada

21

$22 *$ *Correspondence: philips.j.a@wustl.edu 


\section{SUMMARY}

25 Macrophage activation involves metabolic reprogramming to support antimicrobial cellular

26 functions. How these metabolic shifts influence the outcome of infection by intracellular pathogens

27 remains incompletely understood. M. tuberculosis (Mtb) modulates host metabolic pathways and

28 utilizes host nutrients, including cholesterol and fatty acids, to survive within macrophages. We

29 found that intracellular growth of Mtb depends on host fatty acid catabolism: when host fatty acid

$30 \beta$-oxidation (FAO) was blocked chemically with trimetazidine, a compound in clinical use, or

31 genetically by deletion of the mitochondrial fatty acid transporter carnitine palmitoyltransferase 2

32 (CPT2), Mtb failed to grow in macrophages and its growth was attenuated in mice. Global

33 metabolic profiling and mechanistic studies support a model in which inhibition of FAO generates

34 mitochondrial reactive oxygen species, which enhance macrophage NADPH oxidase and

35 xenophagy activity to better control Mtb infection. Thus, FAO inhibition promotes key

36 antimicrobial functions of macrophages and overcomes immune evasion mechanisms of Mtb.

\section{INTRODUCTION}

39 Macrophages are at the forefront of innate immune defense and are required for microbial killing,

40 tissue homeostasis and repair, inflammation, and development. A wealth of scientific studies

41 describes how these cells recognize pathogen-associated molecular patterns (PAMPs), which

42 activate downstream signaling cascades to drive pro-inflammatory responses and resolve

43 infection. Macrophage activation involves profound metabolic reprogramming to support specific

44 phenotypic functions. Exposure of macrophages to stimuli such as IFN- $\gamma$ and LPS induces an

45 inflammatory phenotype characterized by enhanced glycolytic metabolism and impaired oxidative

46 phosphorylation (OXPHOS), similar to the Warburg effect described for cancer cells. Glucose 
47 catabolism provides a rapid means to generate ATP, and boosts the pentose phosphate pathway

48 (PPP) and tricarboxylic acid (TCA) cycle for the generation of important immuno-metabolites

49 such as NADPH, itaconate, and prostaglandins (Galván-Peña and O'Neill, 2014; Kelly and O'Neill,

50 2015; Van den Bossche et al., 2017). On the other hand, cytokines such as IL-4 induce an anti-

51 inflammatory phenotype in macrophages important for tissue homeostasis and anti-parasitic

52 responses. These alternatively activated macrophages have increased FAO and OXPHOS (Galván-

53 Peña and O'Neill, 2014). The majority of studies on macrophage metabolism focus on IFN $\gamma /$ LPS-

54 and IL-4-induced states, although accumulating evidence suggests that macrophage polarization

55 states are multi-dimensional (Xue et al., 2014). The metabolic characterization of more diverse

56 macrophage phenotypes, and their impact on antimicrobial capacity in the context of specific

57 infections is largely unexplored.

59 Recent studies also highlight a link between Mycobacterium tuberculosis (Mtb) pathogenesis and

60 host metabolism. Mtb is the causative agent of tuberculosis (TB), which kills more people yearly

61 than any other infection. Macrophages are a main cellular niche of Mtb (Cohen et al., 2018; Wolf

62 et al., 2007). Within the lung, alveolar (AM) and interstitial (IM) macrophages are the major

63 populations of infected macrophages, and they have distinct metabolic profiles. AMs, which

64 preferentially utilize FAO, represent a permissive niche for Mtb replication, whereas glycolytically

65 active IMs restrict infection (Huang et al., 2018). Mtb alters macrophage metabolism along with

66 shifting macrophage phenotype to a more pro-inflammatory state (Arts et al., 2016; Cumming et

67 al., 2018; Gleeson et al., 2016; Ouimet et al., 2016; Shi et al., 2015). Mtb enhances the dependency

68 of mitochondrial oxidative metabolism on fatty acids, in particular exogenous fatty acids, and

69 induces the formation of lipid-droplet-filled or "foamy" macrophages (Cumming et al., 2018; 
70 Peyron et al., 2008a; Russell et al., 2009; Singh et al., 2012). Foamy macrophages are found within

71 the inner layers of granulomas, a common histopathologic feature of $\mathrm{TB}$, and the bacilli themselves

72 can be found in close approximation to intracellular lipid droplets. It is thought that lipid bodies

73 serve as a source of nutrients in the form of cholesterol esters and fatty acids for the bacilli, thus

74 providing a hospitable niche for the bacterium (Brzostek et al., 2009; Daniel et al., 2011; Marrero

75 et al., 2010; Munoz-Elias and McKinney, 2005; Pandey and Sassetti, 2008; Peyron et al., 2008b;

76 Singh et al., 2012). Moreover, a growing body of literature suggests a link between lipid

77 metabolism and cellular control of Mtb. A number of host-directed therapies (HDTs) under

78 investigation for TB, such as statins and metformin, modulate host lipid metabolism (Parihar et

79 al., 2014; Singhal et al., 2014). However, how these metabolic shifts influence the outcome of Mtb

80 infection remains incompletely understood.

82 Cellular regulators of lipid metabolism such as microRNA-33 (miR-33) and the transcription

83 factors PPAR- $\alpha$ and PPAR- $\gamma$ play a role in the formation of Mtb-induced lipid droplets. Studies in

84 which miR-33, PPAR- $\alpha$, and PPAR- $\gamma$ were modulated revealed a correlation between cellular

85 lipids and intracellular survival of mycobacteria, such that increased intracellular lipids were

86 associated with enhanced bacterial replication (Almeida et al., 2009; Kim et al., 2017; Ouimet et

87 al., 2016). These studies led to the idea that impaired host fatty acid catabolism and the formation

88 of foamy macrophages serves to enhance intracellular survival of Mtb, but direct evidence is

89 lacking. Indeed, miR-33, PPAR- $\alpha$, and PPAR- $\gamma$ impact diverse aspects of host biology that

90 influence the antimicrobial capacity of macrophages, including mitochondrial function and

91 autophagy, and a causal link between the lipid changes they induce and the survival benefit to the

92 bacilli has not been clearly established. Previously, we showed that induction of miR-33/33* in 
93 response to Mtb enhances intracellular survival of Mtb (Ouimet et al., 2016). We showed that this

94 pro-bacterial function of miR-33/33* was related in part to its ability to block autophagy, and we

95 speculated that its ability to block fatty acid catabolism and promote the formation of lipid droplets

96 also enhanced bacterial replication. Here, we tested whether blocking fatty acid catabolism indeed

97 promotes Mtb replication, as prevailing models predict. Instead, we found that inhibiting

98 macrophage FAO chemically or genetically restricted intracellular growth of Mtb. FAO inhibition

99 promoted a pathway of bacterial killing in which induction of mitochondrial ROS (mitoROS) lead

100 to NADPH oxidase and autophagy-dependent control of Mtb.

\section{RESULTS}

103 Inhibiting FAO restricts growth of intracellular Mtb

104 miR-33 inhibits FAO by targeting genes such as carnitine palmitoyltransferase 1 (CPT-1) and the 105 hydroxyacyl-CoA dehydrogenase trifunctional multienzyme complex subunit beta (HADHB).

106 CPT-1 is required for the entry of long chain fatty acids into the mitochondrial matrix, while 107 HADHB catalyzes the final step of $\beta$-oxidation. Small molecule inhibitors, etomoxir (ETM), 108 oxfenicine (OXF), and trimetazidine (TMZ) also target these steps in FAO. ETM and OXF inhibit 109 CPT-1, whereas TMZ blocks the 3-ketoacyl-CoA thiolase activity of HADHB (Figure 1A). Thus, 110 to determine whether the inhibition of FAO conferred by miR-33 contributed to its ability to

111 enhance the intracellular survival of Mtb, we tested the effect of chemical inhibition of FAO on 112 intracellular bacterial replication. We infected murine bone marrow-derived macrophages 113 (BMDMs) with the H37Rv strain of Mtb, and four hours later, we supplemented the media with 114 ETM, OXF, or TMZ. After treatment, we estimated intracellular Mtb growth by plating for colony 115 forming units (CFU) at 72 hours post infection (hpi). Unexpectedly, treatment with all three FAO 
116 inhibitors restricted the intracellular growth of Mtb compared to untreated controls. We observed

117 a dose-dependent reduction in Mtb CFUs in macrophages treated with micromolar concentrations

118 of ETM (Figure 1B), while TMZ and OXF were effective at nanomolar concentrations (Figures

119 1C and D). The antitubercular activity of TMZ was corroborated using a live-dead reporter strain

120 of Mtb (Figure S1A). By comparison, metformin (MET), which has previously been shown to

121 restrict intracellular Mtb, worked at millimolar concentrations (Singhal et al., 2014) (Figures 1B-

122 D). Using calcein fluorescent dye, we found that macrophage viability was unaffected by FAO

123 inhibitors ETM and TMZ (Figure S1B). In addition, the inhibitors did not have direct toxicity on

124 Mtb in broth culture (Figure S1C and S1D), and they enhanced macrophage control against a

125 distantly related mycobacterium, Mycobacterium abscessus (Figure S1E). Consistent with the

126 ability to impair FAO, TMZ enhanced cellular lipid accumulation based upon BODIPY staining

127 (Figure S1F). In addition, in keeping with impaired fatty acid oxidation, TMZ treated

128 macrophages had reduced oxygen consumption (Figure S1G), similar to macrophages from mice

129 that were genetically deficient in FAO due to deletion of carnitine palmitoytransferase $2\left(C p t 2^{f l / f l}\right.$

130 LysM-Cre ${ }^{+}$; Cpt2 cKO) (Gonzalez-Hurtado et al., 2017).

132 To confirm that the anti-mycobacterial activity was indeed a result of FAO inhibition, we

133 compared the intracellular growth of $\mathrm{Mtb}$ in Cpt2 cKO macrophages to littermate controls. As

134 shown in Figure 1E, Mtb was significantly impaired in Cpt2 cKO macrophages as compared to

135 control. Moreover, FAO inhibitors lacked activity in Cpt2 cKO macrophages, confirming their

136 target specificity (Figure 1F). To determine whether FAO inhibitors were also active against Mtb

137 in human macrophages, we treated PMA-differentiated THP-1 cells. As we found in murine

138 macrophages, FAO inhibitors impaired Mtb growth in THP-1 cells (Figure 1G). Taken together, 
139 our findings suggest that FAO inhibition enhances the ability of macrophages to control Mtb

140 infection. Since ETM is documented to have off-target effects (Divakaruni et al., 2018) and TMZ

141 was effective at nanomolar concentrations, we elected it for further study.

\section{Inhibiting FAO restricts growth of Mtb in vivo}

144 Given our in vitro findings, we investigated whether inhibition of FAO impacts mycobacterial control in mice. We compared Mtb infection in mice with myeloid-specific knockout of Cpt2 $\left(C p t 2^{f l f l f l} \mathrm{LysM}^{-\mathrm{Cre}}{ }^{+} ; C p t 2 \mathrm{cKO}\right)$ and littermate controls. We exposed mice to Mtb by aerosol and estimated lung bacterial burden after 7 days. We observed that growth of Mtb was significantly reduced in Cpt2 cKO mice compared to controls (Figure 2A). To determine whether chemical inhibition of FAO had antimicrobial activity in mice, we first performed pharmacokinetic (PK) studies to establish a TMZ dosing strategy (Figure S2A and S2B). The half-life of TMZ in people

151 is approximately 6 hours, but in mice the half-life by oral or IV route was less than 1 hour, so we

152 used Alzet osmotic pumps to deliver a continuous dose over 2 weeks (Figure S2C). First, we

153 examined the ability of TMZ to reduce bacterial burden during acute infection. In the first study

154 we infected mice with 1000-2000 Mtb CFUs and treated them with saline or TMZ for 2 weeks.

155 We found that mice treated with $16.8 \mathrm{mg} / \mathrm{kg} /$ day $\mathrm{TMZ}$ experienced a $67 \%$ reduction in lung

156 bacterial burden compared to animals in the control group (Figure 2B). A significant decrease in 157 spleen CFU was also observed with $16.8 \mathrm{mg} / \mathrm{kg} /$ day $\mathrm{TMZ}$ treatment (Figure 2C). In a second 158 study, we infected mice with 3000 CFUs, tested a higher TMZ dose range, and included PK 159 analysis of infected animals. In this study, $16.8 \mathrm{mg} / \mathrm{kg} /$ day showed a trend towards reduced

160 bacterial growth, which was not statistically significant, while the animals receiving $50 \mathrm{mg} / \mathrm{kg} / \mathrm{day}$ 161 experienced a 29\% reduction in bacterial burden compared to saline controls (Figure 2D and 2E). 
162 In this group, TMZ treatment did not reduce Mtb CFU in spleen. During acute infection, the

163 majority of Mtb bacilli are found within alveolar macrophages, suggesting that TMZ was active in

164 this cell population. As infection progresses, additional phagocytic cells, including recruited 165 monocytes, neutrophils, and dendritic cells become increasingly infected (Cohen et al., 2018; Wolf

166 et al., 2007). To test whether TMZ was active in the setting of chronic infection, we began TMZ

167 treatment 5 weeks post-infection and analyzed bacterial burden after 2 weeks of drug treatment.

168 PK analysis was performed on infected mice 5 days after start of therapy and at end of treatment.

169 In the setting of chronic infection, we found that TMZ treatment reduced bacterial burdens in the

170 lungs by $35 \%$, and in the spleen by $23 \%$ and $43 \%$, in the $16.8 \mathrm{mg} / \mathrm{kg}$ and $50 \mathrm{mg} / \mathrm{kg}$ groups (Figure

1712 F and 2G). TMZ serum concentrations of $130 \mathrm{ng} / \mathrm{ml}$ and $370 \mathrm{ng} / \mathrm{ml}$ were achieved at the end of

172 therapy for the 16.8 and $50 \mathrm{mg} / \mathrm{kg}$ /day groups, respectively (Figure $2 \mathbf{H}$ ). TMZ is approved by the

173 European Medicines Agency to treat angina, and the $\mathrm{C}_{\max }$ obtained after a single $40 \mathrm{mg}$ oral dose

174 in humans is $127 \mathrm{ng} / \mathrm{ml}$. Because there is higher serum protein binding in mice than humans

175 (Figure S2D), the level achieving a similar free drug concentration in mice would be

176 approximately $164 \mathrm{ng} / \mathrm{ml}$. Thus, TMZ showed antimycobacterial activity at drug concentrations

177 that are used clinically. In addition, consistent with the extremely wide therapeutic index of TMZ

178 in preclinical toxicology studies (Harpey et al., 1988), no treatment related morbidity or mortality

179 was observed in any of the studies. Overall, we conclude that inhibiting host fatty acid metabolism

180 restricts mycobacterial growth in vivo.

\section{FAO inhibition enhances metabolic changes in macrophages in response to Mtb infection}

183 We turned to in vitro studies to assess how host fatty acid metabolism was influencing control of

184 Mtb. Given the increasing appreciation of a link between metabolism and immunity, we globally 
185 profiled metabolites to assess the effect of Mtb infection alone or when macrophage FAO was

186 inhibited. Uninfected macrophages or those that had been infected for $4 \mathrm{~h}$ were treated for $3 \mathrm{~h}$ or

187 24h with TMZ or solvent control before profiling (Figure 3A). More than 500 metabolites were

188 detected, and in agreement with previous reports, Mtb infection caused dramatic changes in

189 macrophage metabolites (Figure 3: Figure S3; Table S1); we observed evidence of increased

190 glycolytic and pentose phosphate pathway (PPP) flux (Figure 3B), itaconate production (Figure

191 3C), ROS and reactive nitrogen intermediates (RNI) production (Figure 3DE, 3E and Figure

192 S3A), phospholipid and sphingomyelin changes (Figure S3B), and tryptophan metabolism

193 (Figure S3C). As discussed below, on its own TMZ did not significantly alter metabolic profiles

194 in uninfected macrophages, but in some cases it enhanced infection-induced changes.

196 We observed increases in several glycolytic intermediates (Figure 3B), which suggest augmented

197 glucose catabolism in Mtb-infected macrophages. Early after infection, glucose levels declined in

198 Mtb versus control groups, while glucose-6-phosphate and hexose diphosphates (detected as

199 isobar) accumulated. After $24 \mathrm{~h}$ of Mtb infection, the glucose levels were comparable to uninfected

200 controls, but we observed accumulation in glycolytic intermediates such as phosphoenolpyruvate.

201 The magnitude of responses induced by infection appeared to be greater in TMZ-treated groups.

202 For instance, 24 hpi glucose-6-phosphate was increased 1.52-fold increase by Mtb in drug-exposed

203 cells and 1.12-fold in the absence of TMZ. Glucose can be shunted to the pentose phosphate

204 pathway (PPP) resulting in formation of NADPH reducing power and pentose intermediates for

205 nucleotide biosynthesis. We found that metabolites specific to the PPP, 5-phosphoribosyl

206 diphosphate (PRPP) and sedoheptulose-7-phosphate, increased over time in Mtb-infected 
macrophages, and again, the level of these metabolites was slightly greater upon TMZ treatment

(Figure 3B, Table S1).

210 Classically activated macrophages utilize TCA cycle intermediates for anabolic process and

211 immune pathway signaling. They have a characteristic TCA cycle breakpoint in the conversion of

212 isocitrate to $\alpha$-ketoglutarate. This allows production of itaconate, an antimicrobial metabolite, from

213 cis-aconitate (Figure 3C) (Lampropoulou et al., 2016; Michelucci et al., 2013; Nair et al., 2018).

214 A second TCA cycle breakpoint occurs after succinate. As expected, we observed markedly

215 increased itaconate in infected macrophages, as well as elevated succinate (Figure 3C). There was

216 no substantial difference in the infection-induced elevation in itaconate or succinate in response to

217 TMZ treatment, suggesting these metabolites did not account for the enhanced antimicrobial effect

218 of TMZ.

220 The global metabolomics data also showed evidence of increased oxidative stress in Mtb infected

221 in macrophages relative to uninfected controls, which appeared more pronounced in TMZ-treated

222 samples compared to untreated controls. We observed elevations in citrulline levels early after

223 infection, reflecting the activity of iNOS, which converts arginine to citrulline and NO (Figure

224 3D). This was accompanied by significant increases in dihydrobiopterin and biopterin (Figure

225 S3A). These compounds represent oxidized forms of tetrahydrobiopterin, a cofactor in NO

226 synthesis. Early after infection, the antioxidants glutathione and opthalmate, a compositional

227 derivative of glutathione, were increased by infection, suggesting enhanced glutathione synthetase

228 activity (Figure S3A). This was accompanied by increases in gamma-glutamyl amino acids, which

229 reflect the transfer of gamma-glutamyl moiety of glutathione to acceptor amino acids. (e.g. 
230 gamma-glutamylglutamine) (Figure S3A). After 24 hpi, we observed increases in betaine,

231 dimethylglycine, S-adenosylmethionine (SAM), S-adenosylhomocysteine (SAH) and cystathione

232 (Figure S3A). These shifts could indicate increased methionine to cysteine conversion to support

233 glutathione synthesis. Infected cells appeared to have a more oxidized intracellular milieu after

234 TMZ treatment, as we observed more oxidized glutathione (Figure 3E). Additionally, at the later

235 time point, there were greater infection-induced shifts in SAM, SAH and cystathione levels in

236 infected TMZ-treated cells compared to untreated controls. Combined, the alterations in the

237 glycolytic pathway, itaconate production, and redox homeostasis suggest that macrophages adopt

238 a more M1-like metabolic phenotype in response to Mtb infection, and TMZ-induced perturbations

239 may enhance or prolong these changes, but do not dramatically alter the response.

\section{FAO inhibition triggers mitochondrial ROS to promote mycobacterial control}

242 The metabolomics data did not point to a particular metabolite or metabolic pathway to explain

243 the antimicrobial activity of TMZ. Additionally, we measured the production of TNF- $\alpha$, IL-6,

244 CXCL2, IFN- $\beta$, CCL2, and CXCL10 in response to infection and TMZ treatment, and we did not

245 observe a cytokine-driven antimicrobial response upon TMZ treatment (Figure S4). Since TMZ

246 acts in the mitochondria, a major site of ROS production, and there was evidence of an increase in

247 oxidative stress in TMZ-treated macrophages, we examined whether FAO inhibition promotes

248 ROS production. We treated uninfected immortalized BMDMs (iBMDMs) with TMZ and

249 observed an increase in ROS as early as 3 hours after treatment (Figure 4A). Addition of

250 mitoTEMPO, a mitochondrial ROS (mitoROS) scavenger, abolished this ROS burst, whereas DPI,

251 which inhibits NADPH oxidase had no effect, suggesting that the ROS was induced in the

252 mitochondria (Figure 4A). Toll-like receptor activation and infection with Gram negative bacteria 
253 have previously been shown to result in mitoROS production (Mills et al., 2016; West et al., 2011),

254 and Mtb infection induced a small amount of mitoROS (Figure 4B). However, significantly more

255 ROS was generated in Mtb-infected BMDMs after TMZ treatment (Figure 4C). ROS levels in

256 Mtb-infected Cpt2 cKO BMDMs were also significantly higher than in control macrophages

257 (Figure 4D). The TMZ-induced ROS still occurred in macrophages that lacked the NADPH 258 oxidase (Nox2 KO), consistent with a mitochondrial source (Figure 4C). We confirmed that TMZ

259 induced mitoROS by using MitoSox fluorescent dye (Figure 4E). Combined these results

260 demonstrate that TMZ induces ROS from a mitochondrial source after 3 hours of treatment in both

261 infected and uninfected macrophages.

263 We hypothesized that FAO inhibition resulted in mitoROS generation because of perturbed 264 electron flow within the electron transport chain (ETC). ROS can be generated in multiple sites 265 along the ETC during forward electron transport, as well as when electrons flow in reverse through 266 complex I (NADH:coenzymeQ reductase) (Scialò et al., 2017). To determine the site within the 267 ETC where TMZ induced ROS, we measured mitoROS production in macrophages treated with $268 \mathrm{TMZ}$ in combination with rotenone, an inhibitor of complex I. When electrons are flowing in the 269 forward direction, rotenone prevents electron transport to CoQ, resulting in ROS generation. In 270 contrast during reverse electron transport (RET), rotenone reduces ROS by preventing CoQ from 271 transferring electrons back to complex I, where the RET-ROS is generated. We treated uninfected 272 BMDMs with TMZ for 3h, and thirty minutes prior to measuring mitoROS we added rotenone. As 273 expected, rotenone and TMZ on their own increased mitoROS production. In contrast, rotenone 274 decreased the amount of ROS generated in response to TMZ treatment (Figure 4E) These findings 
275 are consistent with the idea that under conditions of TMZ treatment there is enhanced ROS

276 generated due to RET at complex I.

278 Since recent studies have shown that mitoROS contributes to microbial control in macrophages

279 (Abuaita et al., 2018; West et al., 2011), we asked whether TMZ-induced mitoROS was important

280 for infection control or simply a by-product of metabolic perturbations. To address this, we

281 estimated Mtb burden in macrophages treated with TMZ alone or in combination with

282 mitoTEMPO. As shown in Figure 4F, mitoTEMPO on its own had little effect on the infection,

283 but it partially reversed the antimicrobial effect of TMZ treatment. Overall, we conclude that FAO

284 inhibition promotes mitoROS production from the ETC, which enhances macrophage control of

285 Mtb infection.

FAO inhibition-induced mitochondrial ROS drives NADPH oxidase recruitment to

\section{phagosomes}

289 Previous studies suggest a link between mitoROS and NADPH oxidase that contributes to 290 macrophage defense (Garaude et al., 2016). Indeed, while 3 h of TMZ treatment significantly

291 increased mitoROS in both wt and Nox2 KO BMDMs (Figure 4C), we observed that the anti-Mtb

292 activity of FAO inhibitors required NADPH oxidase (Figure 5A). This suggested that the

293 antimycobacterial activity in FAO-inhibited macrophages actually depended upon both a 294 mitochondrial source and NADPH oxidase. Normally, pathogen associated molecular patterns 295 (PAMPs) promote the recruitment of the NADPH oxidase to microbial phagosomes immediately 296 after phagocytosis (Nunes et al., 2013). However, Mtb impairs the recruitment of the NADPH 
oxidase to the mycobacterial phagosome (Köster et al., 2017; Sun et al., 2013). Remarkably, at 24

hpi we observed that TMZ increased the recruitment of NADPH oxidase subunits gp91 ${ }^{\text {phox}} / \mathrm{NOX} 2$

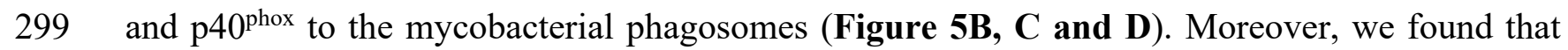

300 NADPH oxidase recruitment was dependent on TMZ-induced mitoROS, as it was reversed with

301 the addition of mitoTEMPO (Figure 5E). Thus, FAO inhibition appears to enhance two

302 antimicrobial responses that are suboptimal during Mtb infection, mitoROS production and

303 phagosomal recruitment of the NADPH oxidase. Mitochondria alone contributed to ROS within

$3043 \mathrm{~h}$ of FAO inhibition, while the role of NADPH oxidase was appreciable at later time points, and

305 macrophage control of Mtb depended upon both mitoROS and the NADPH oxidase.

FAO inhibition promotes xenophagy to restrict intracellular Mtb growth

TMZ treatment in Mtb-infected macrophages resulted in increases in phospholipids and

309 sphingomyelin, which might alter phagosomal trafficking (Figure S3B). In addition, ROS

310 generated by the NADPH oxidase promote a trafficking pathway called LC3-associated

311 phagocytosis (LAP). Both LAP and autophagy of microbes (xenophagy) are characterized by the

312 association of lipidated LC3 (LC3-II) with microbe-containing vacuoles (Martinez et al., 2015).

313 LAP and xenophagy depend upon certain common ATG (autophagy related) proteins, and they

314 also have unique requirements. To determine whether either LC3-trafficking pathway was

315 involved in the antimicrobial activity of TMZ, we compared the efficacy of TMZ between control

316 and Atg5 cKO macrophages, which are deficient in both LAP and xenophagy. Indeed, the

317 antimycobacterial control established by FAO inhibition was reversed in Atg5-deficient

318 macrophages (Figure 6A). Although the baseline ROS staining was lower in Atg5 cKO

319 macrophages compared to control, ROS was still induced by TMZ in Atg5 cKOs, suggesting that 
320 ATG5 acts downstream of mitoROS production (Figure 6B). To distinguish xenophagy from

321 LAP, we examined Atg $14 l \mathrm{cKO}$ and Parkin2 KO macrophages, which are specifically involved in

322 xenophagy. As we had seen in Atg5 cKO macrophages, the antimycobacterial control established

323 by FAO inhibition was reversed in $A t g 14 l \mathrm{cKO}$ and Parkin2 $\mathrm{KO}$ as well (Figure 6C and 6D). In

324 addition, in wt macrophages, TMZ treatment resulted in enhanced co-localization between Mtb

325 and the autophagy adaptor, p62 (Figure 6E). The enhanced colocalization between Mtb and p62

326 occurred after 24 hours treatment and was dependent on mitoROS (Figure 6F and 6G).

327 Combined, these data demonstrate that inhibition of FAO promotes xenophagy to control

328 intracellular Mtb growth. Indeed, autophagosomal targeting of Mtb was also inherently higher in

329 Cpt2 cKO macrophages as compared to wt controls (Figure 6H and 6I). Moreover, TMZ

330 treatment facilitated xenophagy of a mutant Mtb strain lacking esxA $(\operatorname{ses} A)$, which is unable to

331 perforate phagosomes and usually does not activate xenophagy (Figure 6J).

333 These studies established that FAO inhibition induced mitoROS, which promoted NADPH

334 oxidase recruitment to the Mtb phagosome and xenophagy. Next we examined the relationship

335 between the NADPH oxidase and xenophagy. We observed that the enhanced NADPH oxidase

336 recruitment in response to TMZ occurred independently of autophagy, as it was also seen in $\operatorname{Atg} 5$

337 cKO macrophages (Figure 6K). On the other hand, TMZ failed to promote xenophagy in NADPH

338 oxidase-deficient macrophages (Figure 6L). Taken together, our data shows that mitoROS was

339 the primary signal induced by FAO inhibition that resulted in enhanced NADPH oxidase and 340 xenophagy, which promoted bacterial control.

\section{DISCUSSION}


343 Recent work has highlighted the relationship between macrophage metabolism and inflammatory

344 phenotype (Escoll and Buchrieser, 2019). Within the context of Mtb infection, macrophages

345 undergo a Warburg-like shift with enhanced aerobic glycolysis (Gleeson et al., 2016; Lachmandas

346 et al., 2016; Shi et al., 2015) and usage of TCA cycle intermediates for the generation of immuno-

347 metabolites (Nair et al., 2018). Mtb-induced glycolysis reduces intracellular bacterial survival

348 through enhanced IL-1 $\beta$ production, but the impact of fatty acid metabolism is not well established.

349 Mtb also dramatically alter host lipid metabolism, promoting the formation of lipid-droplets

350 (Cumming et al., 2018; Peyron et al., 2008a; Russell et al., 2009; Singh et al., 2012) and increasing

351 mitochondrial dependency on exogenous fatty acids (Cumming et al., 2018). Because Mtb can

352 utilize host fatty acids as a carbon source during infection, a prevailing idea is that host and

353 pathogen are in competition for resources, so that reduced host consumption or enhanced

354 production of cellular lipids would provide a nutrient source for bacterial replication (Almeida et

355 al., 2009; Kim et al., 2017; Ouimet et al., 2016). Instead, we found that inhibition of host FAO

356 impaired mycobacterial replication. Our initial in vitro results are in concordance with a recent

357 report that showed the efficacy of FAO inhibitor ETM in Mtb-infected BMDMs (Huang et al.,

358 2018). In that study, the antimicrobial effect was attributed to reduced IFN- $\beta$, but ETM was used

359 at $200 \mu \mathrm{M}$, which has well-documented off-target effects (Divakaruni et al., 2018). We did not see

360 effects on Type I IFN (Figure S4), and we establish the antimicrobial mechanism of FAO inhibition

361 using specific inhibitors and genetically FAO-deficient Cpt2 knockout macrophages.

362 Unexpectedly, we found that blocking FAO enhanced macrophage effector functions by eliciting

363 mitoROS, which promoted NADPH oxidase and xenophagy to restrict Mtb infection. Our results

364 suggest mitoROS as a connecting link between macrophage fatty acid metabolism and macrophage

365 effector functions to control Mtb infection. 
367 The earliest cellular response that we could detect after FAO inhibition was induction of mitoROS,

368 which occurred within three hours of treatment in both infected and uninfected macrophages.

369 Typically, FAO products, such as $\mathrm{NADH}$ and $\mathrm{FADH}_{2}$, are oxidized by respiratory chain

370 supercomplexes to generate the forward electron flow (FET) required for ATP production. The

371 respiratory chain is organized into interacting supercomplexes to minimize leakage of electrons

372 and ROS formation. Recent studies report that enzymes of FAO also physically interact with ETC

373 components, forming an integrated, multifunctional complex (Wang et al., 2019). The FAO

374 trifunctional protein (TFP), a tetramer that includes the target of TMZ (3-ketoacyl-CoA

375 thiolase/HADHB), interacts with the NADH-binding domain of complex I. The presence of ETC

376 supercomplexes dedicated to FAO may explain why FAO inhibition leads to mitoROS. In the

377 absence of NADH shuttling from FAO to these dedicated supercomplexes, RET might occur. This

378 fits with our finding that combined treatment with TMZ and rotenone, a complex I inhibitor,

379 decreased mitoROS levels, consistent with RET generating the ROS at complex I. mitoROS is

380 also generated in response to microbial products; toll-like receptor signaling alters supercomplex

381 assembly and generates RET-ROS at complex I, both of which impact immunological signaling

382 (Garaude et al., 2016; Langston et al., 2019; Mills et al., 2016; West et al., 2011). Thus, the

383 mitoROS induced by TMZ may activate an antimicrobial pathway that is normally induced and

384 protective in the context of other infection, but suboptimal in during Mtb infection. Interestingly,

385 in distinction to our finding that FAO inhibition generated mROS, the reverse relationship was

386 seen in Salmonella infected zebrafish larvae and J774.2 murine macrophage cells, where FAO

387 enhanced Salmonella-induced mROS (Hall et al., 2013). The difference between this previous 
study and ours could be due to the experimental systems, and it may reflect a different metabolic response of macrophages to mycobacteria as compared to Salmonella.

391 The idea that FAO inhibition enhances macrophage control of Mtb by generating mitoROS is 392 consistent with recent studies showing that mitoROS contributes to macrophage control of 393 Streptococcus pneumonia, Staphylococcus aureus, and Salmonella typhimurium (Abuaita et al., 394 2018; Bewley et al., 2017; Hall et al., 2013; West et al., 2011). Our results suggest that mitoROS 395 does not exert its antimicrobial function by directly killing Mtb, since mitoROS was generated in 396 macrophages lacking Nox2 and Atg5, but the antimycobacterial activity was lost. Rather, mitoROS 397 appears to serve as a signal that enhances NADPH oxidase activity and xenophagy. A stimulatory 398 effect of mitoROS on the NADPH oxidase has been documented most extensively in non399 lymphoid cells, and recent work shows that in neutrophils mitoROS activates the NADPH oxidase 400 and other effector functions (Nazarewicz et al., 2013; Pinegin et al., 2018; Vorobjeva et al., 2017). 401 In addition to promoting the phagosomal recruitment of NADPH oxidase to mycobacterial 402 phagosomes, our metabolomics data indicate that FAO inhibition may augment the PPP, which 403 would enhance NADPH production to power the NADPH oxidase.

405 It will be important to understand how mitoROS promotes phagosomal assembly of the NADPH oxidase. NADPH oxidase assembly requires membrane trafficking of two integral membrane 407 subunits (p22 phox and gp91 phox ) followed by recruitment of the cytosolic subunits (p40 phox, $\mathrm{p} 47^{\text {phox }}$, $408 \mathrm{p} 67^{p h o x}$ ). Normally this occurs shortly after uptake of phagocytic cargo that activate pathogen 409 recognition receptors. In our experimental system, the kinetics are very different than what is 410 typically described. We added TMZ 4h after Mtb had been added to macrophages and when 
411 extracellular bacilli had been washed away. We found that TMZ enhanced localization of both the

412 gp9 $91^{\text {phox }}$ and $\mathrm{p} 40^{\text {phox }}$ subunits with mycobacterial phagosomes 24 hours later. This suggests that

413 signals generated by mitoROS either overcome a Mtb-induced blockade to NADPH oxidase

414 recruitment or promote recruitment through a previously unappreciated pathway. How mitoROS

415 regulates phagosomal NADPH oxidase recruitment and assembly will be important to establish in

416 future studies, as our work indicates that it can be augmented, even after bacterial uptake, to

417 promote microbial clearance of intracellular bacilli. This might be a way to clear foci of persistent

418 bacilli.

420 In addition to NADPH oxidase recruitment, mitoROS also promoted xenophagy. Xenophagy and

421 LAP are related LC3-trafficking pathways that can promote microbial clearance (Upadhyay and

422 Philips, 2019). They also differ, as LAP occurs immediately after phagocytosis and involves LC3

423 recruitment to a single phagosomal membrane, whereas xenophagy depends upon formation of a

424 double membrane compartment. We found that the antimicrobial activity of TMZ depended on

425 ATG14L and PARKIN, which are required for canonical autophagy and Mtb xenophagy

426 (Manzanillo et al., 2013). In addition, TMZ enhanced the association of phagosomes with p62, a

427 selective autophagy adaptor, and the response occurred well after bacterial uptake, all of which

428 suggest that the mechanism of clearance is xenophagy and not LAP, despite the involvement of

429 NADPH oxidase which has been linked to LAP. We also cannot rule out that both LAP and

430 xenophagy are enhanced by FAO inhibition. In terms of how the NADPH oxidase might activate

431 xenophagy, one possibility is that the NADPH oxidase generates phagosomal membrane damage,

432 which is known to promote pathogen ubiquitination, adaptor recruitment, and xenophagy

433 (Manzanillo et al., 2012; Wong and Jacobs, 2011). We observed NADPH oxidase recruitment in 
434 TMZ-treated Atg5 cKO macrophages, yet, TMZ failed to kill Mtb in the absence of xenophagy, 435 arguing against direct antimycobacterial activity of the NADPH oxidase. The lack of direct activity 436 is consistent with other recent studies (Köster et al., 2017; Olive et al., 2018), and likely reflects

437 that Mtb has ROS-detoxifying activities such as KatG, a catalase/peroxidase. We conclude that the 438 antimycobacterial activity of ROS is primarily based upon its ability to activate lysosomal 439 trafficking pathways, perhaps due to damage to the phagosomal membrane resulting in xenophagy 440 activation.

442 While, the current view is that ROS generation contributes to inflammation and cellular damage, 443 a beneficial role of RET-ROS was appreciated recently for hypoxia sensing in the carotid body 444 (Fernández-Agüera et al., 2015), ischemia-reperfusion injury (Chouchani et al., 2014), and 445 extending fly lifespan (Scialò et al., 2016). A number of studies propose the use of FAO inhibitors 446 as metabolic therapy in cancer (Duman et al., 2019; Wang et al., 2018) and heart failure (Lionetti 447 et al., 2011). Given the link that we have shown between FAO inhibition and mitoROS, it will be 448 important to establish whether that contributes to their therapeutic effects. TMZ, which has been 449 used to treat angina for 35 years, is orally bioavailable, lacks substantial drug-drug interactions, 450 and has a favorable safety profile (Dézsi, 2016). Importantly, TMZ showed antimycobacterial 451 activity in mice at drug concentrations that are used clinically. Therefore, TMZ could be rapidly 452 translated into an HDT to be used as an adjunct to conventional antibiotics. The magnitude of Mtb 453 control was modest, but similar to other HDTs that have been tested in mouse models of Mtb 454 (Napier et al., 2011; Parihar et al., 2014; Singhal et al., 2014). In the case of TMZ, the limited 455 activity is probably not due to inadequate drug exposure, but may reflect that TMZ works in only 456 certain myeloid cells in vivo, perhaps based upon their metabolic phenotype, or that there is limited 
457 capacity of mitoROS or autophagy to activate robust clearance. Distinguishing these possibilities

458 will be important in terms of designing effective HDTs for clinical use. Evaluating the effect of

$459 \mathrm{TMZ}$ on other cell types critical for Mtb infection, including neutrophils, dendritic cells, and T

460 cells will be important. Many of the leading candidates for HDT, such as metformin and sirtuin-1,

461 also promote autophagy (Cheng et al., 2017; Singhal et al., 2014), and whether enhanced

462 antimicrobial control could be established by a combination of HDTs that target distinct cellular

463 reservoirs or work synergistically to activate antimicrobial pathways will be critical to investigate.

\section{ACKNOWLEDGEMENTS}

466 We thank members of the Philips laboratory and Robert Mahon (NIH/NIAID) for critical reading

467 of the manuscript. We thank Ronald E. Dolle (Washington University in St. Louis (WUSTL)) for 468 help designing PK studies, Michael J. Wolfgang (Johns Hopkins University School of Medicine)

469 for providing Cpt $2^{f l / f l}$ mice, and Jeff Cox (University of California, Berkeley) for providing Rv-lux 470 strain. We thank the Alvin J. Siteman Cancer Center (WUSTL), Barnes-Jewish Hospital in St 471 Louis, MO, the Bursky Center for Human Immunology and Immunotherapy Programs 472 Immunomonitoring Laboratory, and Diane Bender for the multiplexing immunoassay service. The 473 Siteman Cancer Center is supported in part by an NCI Cancer Center Support Grant \#P30 474 CA091842. This work was supported by grants from the NIH (R21 AI128427), the Center for 475 Drug Discovery (WUSTL), and LEAP Inventor Challenge Award (WUSTL) to J.A.P, NIH (R35 476 HL135799) to K.J.M., NIH (R01 DK11003404) to J.D.S., and the Pott's Memorial Foundation to 477 P.C. 


\section{AUTHOR CONTRIBUTIONS}

480 M.O. and S.K. did experiments demonstrating activity of etomoxir against intracellular Mtb and

481 P.C. performed all subsequent experiments. She had help with mice and MIC measurements from

482 G. Y. and SeaHorse experiments by L.H. M.Z., H.W., and V.D. measured TMZ concentrations

483 from serum of infected mice. J.A.P. conceived the project and supervised the experiments with

484 input from J.D.S. P.C. and J.A.P. wrote the manuscript; P.C., S.K., M.O., K.J.M., V.D., J.D.S, 485 and J.A.P. edited the manuscript.

486

DECLARATION OF INTERESTS

488 The authors declare no competing interests.

489

490

491

492

493

494

495

496

497

498 
STAR Methods

500 KEY RESOURCES TABLE

\begin{tabular}{|c|c|c|}
\hline REAGENT or RESOURCE & SOURCE & IDENTIFIER \\
\hline \multicolumn{3}{|l|}{ Antibodies } \\
\hline gp91 phox antibody & Abcam & Cat\# ab80508 \\
\hline p40-phox (N-20-R) antibody & Santa Cruz Biotechnology & Cat\# sc-18252-R \\
\hline $\begin{array}{l}\text { Human/Mouse/Rat p62/SQSTM1 } \\
\text { Alexa Fluor } 488 \text { conjugated } \\
\text { antibody }\end{array}$ & R\&D systems & Cat\# IC8028G \\
\hline $\begin{array}{l}\text { Alexa Fluor } 488 \text { goat and rabbit } \\
\operatorname{IgG}(\mathrm{H}+\mathrm{L})\end{array}$ & Thermo Fisher Scientific & Cat\#A11034 \\
\hline \multicolumn{3}{|l|}{ Chemicals and Inhibitors } \\
\hline Etomoxir & Tocris Bioscience & Cat\# 4539 \\
\hline Trimetazidine & Cayman Chemical Company & Cat\# 18165 \\
\hline Oxfenicine & Sigma Aldrich & Cat\# 56160-10G \\
\hline Metformin & Sigma Aldrich & Cat\# D150959-5G \\
\hline mitoTEMPO & Sigma Aldrich & Cat\# SML0737-5MG \\
\hline Amikacin & Sigma Aldrich & Cat\# A1774 \\
\hline BODIPY 493/503 & Thermo Fisher Scientific & Cat\# D3922 \\
\hline Calcein AM cell permeant dye & Thermo Fisher Scientific & Cat\# C1430 \\
\hline $\begin{array}{l}\text { Diphenyleneiodonium chloride } \\
\text { (DPI) }\end{array}$ & Sigma Aldrich & Cat\# D2926-10MG \\
\hline Tert-Butyl hydroperoxide (tBHP) & Sigma Aldrich & Cat\# 416665-100ML \\
\hline $\mathrm{N}$-acetyl L-cysteine (NAC) & Sigma Aldrich & Cat\# A7250-5G \\
\hline Rotenone & Sigma Aldrich & Cat\#R8875 \\
\hline Antimycin & Sigma Aldrich & Cat\#A8674 \\
\hline Menadione & Sigma Aldrich & Cat\# M5625 \\
\hline Oligomycin & Sigma Aldrich & Cat\#C2920 \\
\hline FCCP & Sigma Aldrich & Cat\# P8139 \\
\hline $\begin{array}{l}\text { Phorbol 12-myristate 13-acetate } \\
\text { (PMA) }\end{array}$ & Sigma Aldrich & Cat\# P8139 \\
\hline CellRox Green Reagent & Thermo Fisher Scientific & Cat\# C10444 \\
\hline $\begin{array}{l}\text { MitoSox Red mitochondrial } \\
\text { superoxide indicator }\end{array}$ & Thermo Fisher Scientific & Cat\# M36008 \\
\hline \multicolumn{3}{|l|}{ Deposited Data } \\
\hline N/A & & \\
\hline \multicolumn{3}{|l|}{ Commercial Assays } \\
\hline $\begin{array}{l}\text { Milliplex MAP Mouse } \\
\text { Cytokine/Chemokine Magnetic } \\
\text { Bead Panel }\end{array}$ & EMD-Millipore & Cat\# MCYTOMAG-70K \\
\hline $\begin{array}{l}\text { IFN-alpha/IFN-beta 2-plex Mouse } \\
\text { ProcartaPlex Panel }\end{array}$ & Thermo Fisher Scientific & Cat\# EPX02A-22187-901 \\
\hline \multicolumn{3}{|l|}{ Software and Algorithms } \\
\hline GraphPad Prism & & www.graphpad.com \\
\hline
\end{tabular}




\begin{tabular}{|l|l|l|}
\hline \multicolumn{2}{|l|}{ NIS Elements version 4.40 } & \multicolumn{2}{|l|}{} \\
\hline \multicolumn{2}{|l|}{ Culture supplies and supplements } \\
\hline $\begin{array}{l}\text { Dulbecco's Modified Eagle Medium } \\
\text { (DMEM) }\end{array}$ & Thermo Fisher Scientific & Cat\# 11995065 \\
\hline RPMI 1640 Medium & Thermo Fisher Scientific & Cat\# 11875093 \\
\hline Fetal bovine serum (FBS) & Thermo Fisher Scientific & Cat\# 26140079 \\
\hline BD Difco Middlebrook 7H9 Broth & BD Biosciences & Cat\# 271310 \\
\hline $\begin{array}{l}\text { BD BBL Middlebrook ADC } \\
\text { Enrichment }\end{array}$ & BD Biosciences & Cat\# 212352 \\
\hline BD Difco Middlebrook 7H11 Agar & BD Biosciences & Cat\# 283810 \\
\hline $\begin{array}{l}\text { BD BBL Middlebrook OADC } \\
\text { Enrichment }\end{array}$ & BD Biosciences & Cat\# 212351 \\
\hline
\end{tabular}

502 LEAD CONTACT AND MATERIALS AVAILABILITY

503 Further information and requests for reagents may be directed to and will be fulfilled by the Lead

504 Contact, J. A. Philips (philips.j.a@wustl.edu)

\section{EXPERIMENTAL MODEL DETAILS}

\section{Bacterial Strains}

$511 \mu \mathrm{g} / \mathrm{ml}$ hygromycin depending on the resistance marker. H37Rv, the wild type Mtb strain, and

$512 \Delta e s x A$ were provided by William Jacobs Jr. (Albert Einstein College of Medicine)(Wong and

513 Jacobs, 2011). DsRed expressing H37Rv was a gift from J. Ernst (New York University). H37Rv

514 expressing the Vibrio harvei luciferase (Rv-lux) was gift from Jeff Cox (University of California,

515 Berkeley). The "live-dead" reporter strain constitutively expresses mCherry and inducibly express

516 GFP under control of a tetracycline-inducible promoter (Martin et al., 2012). 


\section{Mice}

519 We used 8-12 week old C57BL/6 mice. Cpt $2^{f l f l}$ LysM-Cre $^{+}$mice were generated by crossing $520 C p t 2^{f l f l}$ and LysM-Cre ${ }^{+}$mice. The generation of $C p t 2^{f l f l}$ and LysM-Cre ${ }^{+}$mice have been described

521 previously (Clausen et al., 1999; Lee et al., 2015). The Washington University School of Medicine

522 Institutional Animal Care and Use Committee approved all the work with mice. Euthanasia was

523 performed prior to bone marrow harvest in accordance with the 2013 AVMA Guidelines for the

524 Euthansia of Animals (https://www.avma.org/KB/Policies/Documents/euthanasia.pdf).

\section{METHOD DETAILS}

\section{Cell culture}

528 To obtain murine bone marrow-derived macrophages (BMDMs), marrow was flushed from the 529 femurs and tibia of mice, and the hematopoetic stem cells were allowed to differentiate for 7 days

530 in Dulbecco's Modified Eagle Medium (DMEM, Gibco) supplemented with 10\% fetal bovine 531 serum (FBS, Gibco), 1\% Pen-Strep solution (Gibco), and 20\% L929-conditioned medium. After

5327 days, the BMDMs were harvested using $\mathrm{Ca}^{2+} / \mathrm{Mg}^{2+}$-free PBS (Gibco) containing 5mM EDTA

533 (Invitrogen, Life Technologies), and maintained in DMEM containing 10\% FBS and 10\% L929-

534 conditioned medium after infection. Immortalized BMDMs (iBMDMs) were immortalized by 535 infection with the J2 retrovirus (BEI Resources). RAW 264.7 and THP-1 cells were obtained from 536 American Type Tissue Collection (ATCC) and were maintained in DMEM and RPMI-1640, 537 respectively, with 10\%FBS. THP-1 differentiation was induced using $20 \mathrm{ng} / \mathrm{ml}$ phorbol-12538 myristate acetate (PMA, Sigma) for 18-20 hours. 


\section{Bacterial infections}

541 For in vitro macrophage assays, a log phase culture of Mtb H37Rv was pelleted and resuspended

542 in macrophage culture medium. Bacterial single-cell suspensions were prepared by filtering

543 through 5-micron filters (PALL Life Sciences, catalog no. 4650). The number of Mtb in the

544 resultant filtrate was estimated by measuring absorbance at $600 \mathrm{~nm}$, followed by infection of

545 macrophages at a multiplicity of infection (MOI) of 5. After 4 hours, macrophages were washed

546 three times with warm DMEM to remove extracellular bacteria, and then resuspended in medium

547 containing FAO inhibitors or solvent control. To estimate intracellular Mtb growth, infected

548 macrophages were lysed in $0.06 \%$ SDS solution at the indicated time points, and serial dilutions

549 of the lysates were plated on 7H11 agar plates (BD Biosciences, catalog no. 283810) containing

550 BD BBL Middlebrook OADC Enrichment (BD Biosciences, catalog no. 212351) and glycerol.

551 Colony forming units (CFU) were calculated 14-21 days later. For in vivo infections, log phase

$552 \mathrm{H} 37 \mathrm{Rv}$ culture was pelleted and resuspended in sterile $0.5 \%$ Tween 80 solution. After a

553 centrifugation step, the supernatant was used for aerosol infection using a Glas-Col inhalation

554 exposure system. The infectious dose administered was calculated by plating CFU from an aliquot

555 of the bacterial suspension.

\section{Mice surgeries and aerosol infections}

558 Alzet mini-osmotic pumps (Model 2002, Durect Corporation, CA) were loaded with saline or TMZ

559 solution as per the manufacturer's protocol. The osmotic pumps were surgically implanted in

560 anesthetized mice under aseptic conditions. The mice were administered analgesic to minimize

561 pain and were monitored regularly for signs of pain and other postoperative complications. Two

562 days later, the mice were infected with $\mathrm{H} 37 \mathrm{Rv}$ via aerosol route using an inhalation exposure 
563 system from Glas-Col. The dose of infection was confirmed one day post-infection by plating

564 whole lung homogenates from 2 mice on Middlebrook 7H11 agar. 2 weeks post infection, the mice

565 were euthanized and the lungs were harvested, homogenized, and plated for CFUs.

567 Serum microsampling of TMZ

568 Serum concentrations of TMZ in mice were determined at 5 and 14 days after initiating treatment.

569 Lidocaine was applied to mice tails to minimize pain, and the end of the tail was wiped with alcohol

570 and a small incision was made. $100 \mu \mathrm{l}$ blood was collected in $\mathrm{K}_{2}$-EDTA microvette tubes

571 (Braintree Scientific, Inc) and was centrifuged at 5,000 rpm for 5 minutes to recover plasma.

572 Samples were stored at $-80^{\circ} \mathrm{C}$ until analyzed for $\mathrm{TMZ}$ content.

High pressure liquid chromatography coupled to tandem mass spectrometry (LC/MS-MS)

$5761 \mathrm{mg} / \mathrm{mL}$ DMSO stocks of Trimetazidine (Sigma-Aldrich) were serial diluted in 50/50 Acetonitrile

577 water and subsequently serial diluted in drug free CD1 mouse plasma $\left(\mathrm{K}_{2}\right.$ EDTA, Bioreclamation

578 IVT, NY) to create standard curves and quality control (QC) spiking solutions. Standards, QC,

579 controls, and study samples were extracted by combining $10 \mu \mathrm{L}$ plasma with $100 \mu \mathrm{L}$

580 acetonitrile/methanol 50/50 containing the internal standard verapamil. Extracts were vortexed

581 and centrifuged, and supernatant was transferred for LC-MS/MS analysis. LC/MS-MS was

582 performed on a Sciex Applied Biosystems Qtrap 6500+ triple-quadrupole mass spectrometer

583 coupled to a Shimadzu 30ACMP HPLC system, and chromatography was performed on an Agilent

584 Zorbax XDB-C 18 column $(3 \times 75 \mathrm{~mm}$; particle size, $3.5 \mu \mathrm{m})$. Milli-Q deionized water with $0.1 \%$ 
585 formic acid was used for the aqueous mobile phase and $0.1 \%$ formic acid in acetonitrile for the

586 organic mobile phase. Multiple-reaction monitoring (MRM) of parent/daughter transitions in

587 electrospray positive-ionization mode was used to quantify all molecules. MRM transitions of

588 267.08/181.10 and 455.40/165.20 were used for TMZ and Verapamil respectively.

590 Fluorescence microscopy and image analyses

591 BMDMs were seeded in 8-well chamber slides (Falcon culture slide 8-well, catalog no. 08-774-

592 26), and infected with DsRed-expressing H37Rv at MOI 5. At the indicated time points, samples

593 were fixed overnight with 1\% paraformaldehyde (PFA). For immunofluorescence (IF), samples

594 were permeabilized and blocked in PBS with $0.05 \%$ saponin and 3\% BSA, and stained with the

595 indicated primary antibodies for $2 \mathrm{~h}$ at room temperature or overnight at $4^{\circ} \mathrm{C}$. Primary antibodies

596 used were p40 phox (Santa Cruz Biotechnologies), gp91 phox/NOX2 (abcam), and p62 (R\&D

597 systems). Staining with alexa fluorophore-conjugated secondary antibody (Molecular Probes) was

598 done for $2 \mathrm{~h}$ at room temperature. Following this, the samples were washed with $0.1 \%$ Tween

599 20/PBS and mounted using Prolong Gold antifade (Thermo Fisher Scientific, catalog no. P36930).

600 Images were captured using a Nikon Eclipse Ti confocal microscope (Nikon Instruments Inc.)

601 equipped with a 60X apochromat oil-objective lens, and analyzed using NIS-Elements version

6024.40 (Nikon). Briefly, a region of interest (ROI) was drawn around each bacterium and the mean

603 fluorescence intensity (MFI) was measured using the ROI statistics tool.

604

605 ROS measurement assays 
606 For ROS measurement assays, the macrophages were seeded in 96 well plates ( $\mu$-Plate 96 well,

607 IBIDI catalog no. 89626). To estimate total cell ROS, the samples were treated with Cell Rox

608 green fluorescent dye (Thermo Fisher Scientific) at $5 \mu \mathrm{M}$ for 30 minutes. The samples were

609 washed three times with PBS, and fixed overnight with 1\% PFA. Mitochondrial ROS was

610 measured in live, uninfected macrophages using MitoSox fluorescent dye (Thermo Fisher

611 Scientific), at $5 \mu \mathrm{M}$ for 30 minutes. The samples were imaged using confocal microscope, and the

612 ROS signal of each cell was quantified using NIS-Elements software. Briefly, each cell was

613 converted to a ROI and the MFI was measured using the ROI statistics tool.

\section{Oxygen consumption rate and extracellular acidification rate}

616 BMDMs from Cpt $2 \mathrm{cKO}$ mice and control littermates were plated in 96 well Seahorse plates at a

617 density of 75,000 cells per well. The cells were treated with 5nM TMZ or solvent control for $3 \mathrm{~h}$.

618 After treatment, the cells were washed and placed in XF media (non-buffered RPMI 1640

619 containing $25 \mathrm{mM}$ glucose, $2 \mathrm{mM}$ L-glutamine and $1 \mathrm{mM}$ sodium pyruvate) with $10 \%$ FCS. Oxygen

620 consumption rate (OCR) and extracellular acidification rates (ECAR) were measured under basal

621 conditions and following inhibitors were added: $1 \mu \mathrm{M}$ oligomycin (Sigma), $1.5 \mu \mathrm{M}$ fluorocarbonyl

622 cyanide phenylhydrazone (FCCP, Sigma), and $100 \mathrm{nM}$ rotenone (Sigma) $+1 \mu \mathrm{M}$ antimycin A

623 (Sigma). Measurements were taken using a 96 well Extracellular Flux Analyzer (Seahorse

624 Bioscience, North Bellerica, MA, USA).

625 
627 For metabolic profiling of murine BMDMs, 15 million macrophages were seeded in $15 \mathrm{~cm}$ petri

628 dishes (Genesee Scientific) with 5 replicates for each condition. The next day, macrophages were

629 infected with $\mathrm{H} 37 \mathrm{Rv}$ at an MOI 5 for $4 \mathrm{~h}$ as described above. The samples were washed and

630 maintained in culture medium with or without with $50 \mathrm{nM} \mathrm{TMZ}$ for 3 or $24 \mathrm{~h}$. At the respective

631 time points, the samples were washed twice with sterile Hank's Balanced Salt Solution (HBSS,

632 Gibco), and the metabolites were extracted in $80 \%$ methanol (Sigma) in water (Corning). The

633 samples were stored at $-80^{\circ} \mathrm{C}$ and shipped to Metabolon Inc., $\mathrm{NC}$ (www.metabolon.com) for

634 further processing and analyses.

635 At Metabolon, the samples were prepared using the automated MicroLab STAR ${ }^{\circledR}$ system from

636 Hamilton Company. After addition of recovery standards and protein removal, the extracts were

637 divided into fractions for analysis by: two separate reverse phase (RP)/ UPLC-MS/MS with

638 positive ion mode electrospray ionization (ESI), RP/UPLC-MS/MS with negative ion mode (ESI),

639 and HILIC/UPLC-MS/MS with negative ion mode ESI. All methods utilized a Waters ACQUITY

640 ultra-performance liquid chromatography (UPLC) and a Thermo Scientific Q-Exactive high

641 resolution/accurate mass spectrometer interfaced with a heated electrospray ionization (HESI-II)

642 source and Orbitrap mass analyzer operated at 35,000 mass resolution.

\section{Cytokine measurements}

645 Culture supernatants were harvested from uninfected or Mtb-infected BMDMs that were untreated

646 or treated with 5 or $50 \mathrm{nM} \mathrm{TMZ}$ for 24 and $72 \mathrm{~h}$. The conditioned media was filter-sterilized and

647 cytokines and chemokines were measured using Milliplex MAP Mouse Cytokine/Chemokine

648 Magnetic Bead Panel (MCYTOMAG-70K, Millipore Sigma) and Procartaplex Mouse IFN $\alpha /$ IFN $\beta$

649 Panel (2plex) (ThermoFisher Scientific). 


\section{Liquid MIC determinations}

652 In 96 well plates, Mtb cultures with starting $\mathrm{OD}_{600}$ of $0.01,0.05$, and 0.10 were incubated with

653 increasing concentrations of drugs in triplicate. The growth of Mtb was measured at day $0,1,2,3$

654 and 4. The MIC was considered the minimal concentration tested that inhibited Mtb growth at 4

655 days. To assess the direct toxicity of TMZ on Mtb, H37Rv expressing the Vibrio harvei luciferase

656 (Rv-lux) was cultured in $7 \mathrm{H} 9$ broth with or without $1 \mathrm{mM}$ TMZ for 7 days. Relative luminescence

657 units (RLU) were measured every 2 days at $490 \mathrm{~nm}$.

\section{PK studies}

660 PK and plasma binding studies were performed by Alliance Pharma (PA, USA) following IV and 661 oral administration of 3 and $15 \mathrm{mg} / \mathrm{kg}$ TMZ, respectively. PK study of TMZ using Alzet osmotic 662 pumps was conducted by Paraza Pharma Inc, Canada. Briefly, Alzet osmotic pumps (model 2002, 663 Durect Corporation) were surgically implanted in C57BL/6 mice $(\mathrm{n}=5)$ for a subcutaneous infusion 664 at $10.66 \mathrm{~m} / \mathrm{k} /$ day. Plasma TMZ concentrations were determined over the course of $48 \mathrm{~h}$.

666 Statistics

667 GraphPad Prism software was used to prepare plots and assess statistical significance of results 668 using unpaired Student's t-test with Welch's correction, Ordinary one-way ANOVA and Mann669 Whitney test. 


\section{FIGURE LEGENDS}

\section{Figure 1. Inhibition of macrophage FAO restricts intracellular Mtb growth.}

674 (A) In the carnitine shuttle, long chain fatty acids that have been activated to acyl-CoA derivatives

675 are converted to acylcarnitines by CPT1 at the outer mitochondrial membrane. Etomoxir and

676 oxfenicine inhibit CPT1. Acylcarnitines are transported across the mitochondrial membrane by a

677 dedicated translocase. In the mitochondria, CPT2 converts acylcarnitines back to acyl-CoA and

678 carnitine. Acyl-CoA chains then undergo $\beta$-oxidation, successively generating acetyl-CoA that

679 enters the TCA cycle. Trimetazidine inhibits 3-ketoacyl-CoA thiolase, which catalyzes the release

680 of acetyl-CoA from the acyl-CoA chain. (B-D) Survival of Mtb (H37Rv) in BMDMs that were

681 untreated or treated for $72 \mathrm{~h}$ with $2 \mathrm{mM}$ metformin (MET) or indicated concentrations of ETM

682 (B), TMZ (C), or OXF (D). (E) Survival of Mtb in BMDMs from Cpt2 KO (Cpt ${ }^{f l f l}$ LysM-Cre $^{+}$)

683 and littermate controls $\left(C p t 2^{f l f f} \mathrm{Cre}^{-}, \mathrm{WT}\right)$ 4, 72 and 120 hpi. (F) Survival of Mtb in BMDMs from

$684 C p t 2 \mathrm{cKO}$ and WT controls treated with ETM, TMZ, or vehicle control for $72 \mathrm{~h}$. CFU are expressed

685 as \% of WT untreated control. (G) PMA-differentiated THP-1 cells were infected with Mtb and

686 treated with ETM or TMZ for 72 hpi prior to enumerating CFU. (B-G) Data shows mean +/- s.e.m.

687 from one representative experiment from at least 2 independent experiments. * ${ }^{*} \leq 0.009$,

$688 * * * \mathrm{P}=0.0003, * * * * \mathrm{P}<0.0001, \mathrm{NS}-$ not significant, one-way ANOVA (B-D, F-G) or unpaired

689 Students t-test with Welch's correction (E). See also Figure S1.

\section{Figure 2. FAO inhibition restricts growth of Mtb in vivo}

692 (A) Cpt2 cKO mice and littermate controls were infected by aerosol with 100-200 Mtb CFU per

693 animal, and CFU were enumerated from lungs 7 days post-infection. $* \mathrm{P}=0.04$, (B-D) Efficacy of

694 TMZ treatment was tested in mice that were acutely infected with Mtb. Plots show total lung and 
695 spleen CFU for the first study (B, C) and total lung CFU for the second study (D) after 2 weeks of

$696 \mathrm{TMZ}$ treatment. $* \mathrm{P}=0.03, * * \mathrm{P}=0.02, * * * \mathrm{P}=0.0002$. (E) Serum levels of $\mathrm{TMZ}$ were measured at

697 the end of therapy in mice shown in (D). (F,G) In the chronic study, mice that were infected by

698 aerosol with Mtb for five weeks were treated with TMZ or saline control for 2 weeks (shown in

699 gray region) and total lung and spleen $\mathrm{CFU}$ were enumerated. $* \mathrm{P}=0.01, * * \mathrm{P} \leq 0.005, * * * \mathrm{P}=0.0002$.

700 (H) Serum levels of TMZ were measured in mice shown in (F) after 5 days of starting treatment

701 ( $\Delta$ ) and at the end of therapy (०). Data shows mean +/- s.e.m., P-values calculated using Mann-

702 Whitney rank sum test. See also Figure S2.

Figure 3. FAO inhibition enhances metabolic changes in response to Mtb infection.

705 (A) Global metabolite profiling was performed on uninfected or Mtb infected BMDMs to assess

706 the effect of infection alone or after treatment with 50nM TMZ for 3 or $24 \mathrm{~h}$. (B) Macrophage

707 glycolysis was augmented in response to Mtb infection, and a sharp accumulation in glycolytic

708 intermediates was observed. Glucose-6-phosphate was shunted to the pentose phosphate pathway

709 for the production of PRPP and sedoheptulose-6-phosphate. The pathway heat map shows

710 metabolite ratios in Mtb infected and uninfected (TB/Ctrl) macrophages, that were untreated

711 (None) or TMZ treated (Drug) for 3 or $24 \mathrm{~h}$. Here, significant difference $(\mathrm{p} \leq 0.05$, three-way

712 ANOVA) between groups are colored in green for metabolite ratio of $<1$ and red for ratio of $>1$.

713 Light green and red colors show groups that narrowly missed statistical cutoff for significance

$7140.05<\mathrm{p}<0.10$. (C) Mtb infection induced levels of intermediates itaconate and succinate. This

715 reflected breakpoints in the TCA cycle that are characteristic of inflammatory macrophage

716 phenotype. Innate immune response of macrophage against the pathogen manifested as elevations

717 in (D) citrulline, an iNOS by product, and (E) oxidized glutathione, a host redox metabolite. (C- 

samples at the indicated time points. The line and dot show data median and mean, respectively.

\section{Figure 4. FAO inhibition induces mitochondrial ROS burst required for Mtb killing.}

723 CellRox mean fluorescence intensity (MFI) of (A) uninfected iBMDMs that were untreated or treated with 500nM TMZ for $3 \mathrm{~h}$, alone or in presence of mitoTEMPO $(10 \mu \mathrm{M})$ or DPI $(10 \mathrm{mM})$,

727 infected Cpt2 $\mathrm{cKO}$ and control BMDMs 24 hpi that were untreated or treated with N-acetyl 728 cysteine (NAC, 10mM). tert-Butyl hydroperoxide (tBHP, $0.25 \mathrm{mM}, 30 \mathrm{mins}$ ) was used as a positive 729 control. $* \mathrm{P}=0.02, * * \mathrm{P}=0.001, * * * * \mathrm{P}<0.0001$, NS- not significant, calculated using unpaired

730 Student's t-test with Welch's correction (A) and ordinary one-way ANOVA (B-D). (E) MitoSox

731 MFI of uninfected BMDMs that were untreated or treated with 500nM TMZ for 3h, alone or in 732 presence of rotenone $(10 \mu \mathrm{M}, 30 \mathrm{mins}) . * \mathrm{P}=0.02, * * * * \mathrm{P} \leq 0.0001$, ordinary one-way ANOVA. (F)

733 Mtb CFU in BMDMs that were untreated or treated with 50nM TMZ alone or in combination with 734 mitoTEMPO for $120 \mathrm{hr}$. Data from one of 2 independent experiments shows mean +/735 s.e.m. ${ }^{*} \mathrm{P}=0.005$ calculated using unpaired Student's t-test with Welch's correction. (A-E) Data

736 shown is mean +/- s.e.m. of MFI derived from individual cells in the sample. At least 100 cells 737 were analyzed from duplicate samples. For (B, E), values are expressed as \%uninfected.

\section{$738 \quad$ See also Figure S4.}


Figure 5. FAO inhibition promotes NADPH oxidase recruitment on phagosomes.

741 (A) Survival of Mtb in BMDMs from Nox2 KO and control mice that were untreated or treated 742 with indicated concentrations of ETM and TMZ, 120 hpi. $* \mathrm{P}=0.04$, $* * * * \mathrm{P}=0.0001$, ordinary one-

743 way ANOVA. (B) Immunofluorescence (IF) microscopy of gp91 phox/NOX2 (green) and dsRed-

744 expressing H37Rv (red) in BMDMs that were infected and treated with 5nM TMZ or untreated

745 for $24 \mathrm{~h}$. The co-localized region is shown in yellow, scale bar $=10 \mu \mathrm{m}$. MFI of the NADPH

746 oxidase subunits (C) gp91 phox $/ \mathrm{NOX} 2$ and (D) p40 phox co-localized with H37Rv in BMDMs that

747 were untreated or treated with 5nM TMZ (24hpi). (E) MFI of p40 phox co-localized with Mtb in

748 BMDMs treated with 5nM TMZ alone or in presence of mitoTEMPO (48 hpi). (C-E) Automated

749 image analysis was used to quantify gp91 phox/NOX2 or $\mathrm{p} 40^{\text {phox }}$ MFI co-localized with over 100

750 bacilli in at least 2 independent experiments. Data shows mean $+/-$ s.e.m. of MFI from around

751 individual bacilli in the sample. $* \mathrm{P}=0.02, * * * * \mathrm{P} \leq 0.0001$ calculated using unpaired Student's t-test

752 with Welch's correction (C-D) or ordinary one-way ANOVA (E).

Figure 6. Antimicrobial activity of FAO inhibitors depends on autophagy.

755 (A) Survival of Mtb was compared in FAO inhibitor treated control and Atg5 cKO macrophages,

756 72hpi. (B) CellRox MFI in Mtb infected control and Atg5 cKO BMDMs after TMZ treatment (5 $757 \mathrm{nM}, 3 \mathrm{hr}$ ), with or without mitoTEMPO. $* * * * \mathrm{P} \leq 0.0001$, unpaired Student's t-test with Welch's

758 correction. Survival of Mtb in BMDMs from control and (C) Atg14l cKO cells 72 hpi, and (D)

759 Parkin2 KO cells 120 hpi. Data shows mean +/- s.e.m. *P=0.04, using unpaired t-test with Welch's

760 correction in $(\mathrm{A})$ or $* * * \mathrm{P}=0.0002, * * * * \mathrm{P} \leq 0.0001$ using ordinary one-way ANOVA in (C, D). (E)

761 IF microscopy of p62 (green) and dsRed-expressing H37Rv (red) in BMDMs treated with 5nM

762 TMZ 24hpi. The co-localized region is shown in yellow, scale bar $=10 \mu \mathrm{m}$. MFI of p62 co- 
763 localized with Mtb was measured in BMDMs treated with 5nM TMZ alone or with mitoTEMPO

764 for (F) 3 or (G) 24 hours. $* * * * \mathrm{P} \leq 0.0001$, ordinary one-way ANOVA. (H) IF images and (I) MFI

765 of p62 co-localized with Mtb in Cpt2 KO and control BMDMs 24hpi. (J) MFI of p62 co-localized

766 with $\triangle e s x A$ in BMDMs treated with TMZ for 24h. (K) MFI of gp91 phox/NOX2 co-localized with

767 Mtb in control versus Atg5 cKO BMDMs after TMZ treatment (5nM, 24hpi). (L) MFI of p62 co-

768 localized with Mtb in control and Nox2KO macrophages treated with 5nM TMZ with or without

769 mitoTEMPO, 24 hpi. Data shows mean + - s.e.m. $* * \mathrm{P}=0.001$, **** $\mathrm{P} \leq 0.0001$ calculated using

770 unpaired Student's t-test with Welch's correction in $(\mathrm{K})$ and ordinary one-way ANOVA for (J,L).

771 All panels show data from one representative experiment from at least 2 independent replicates.

772

773

774

775

776

777

778

779

780

781

782

783 
A
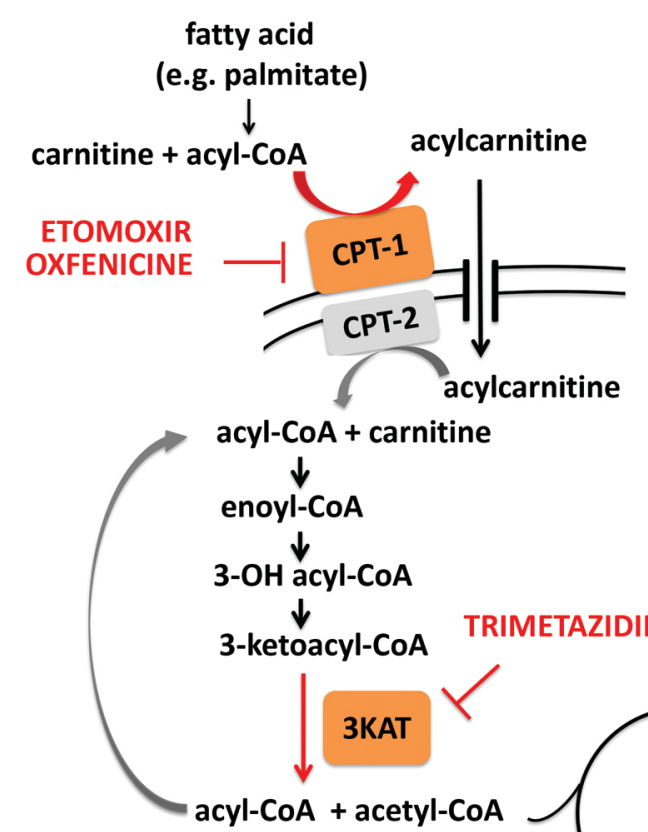

D

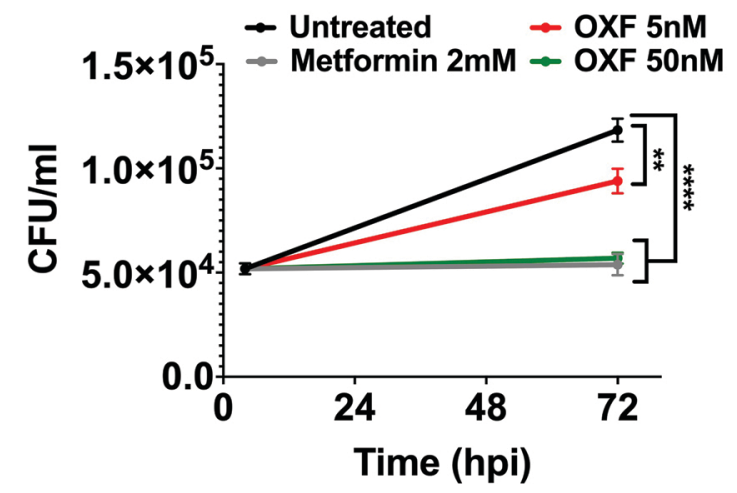

$\mathbf{F}$

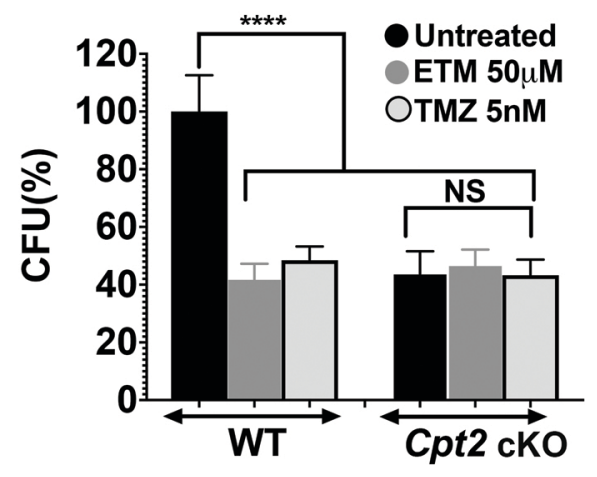

B
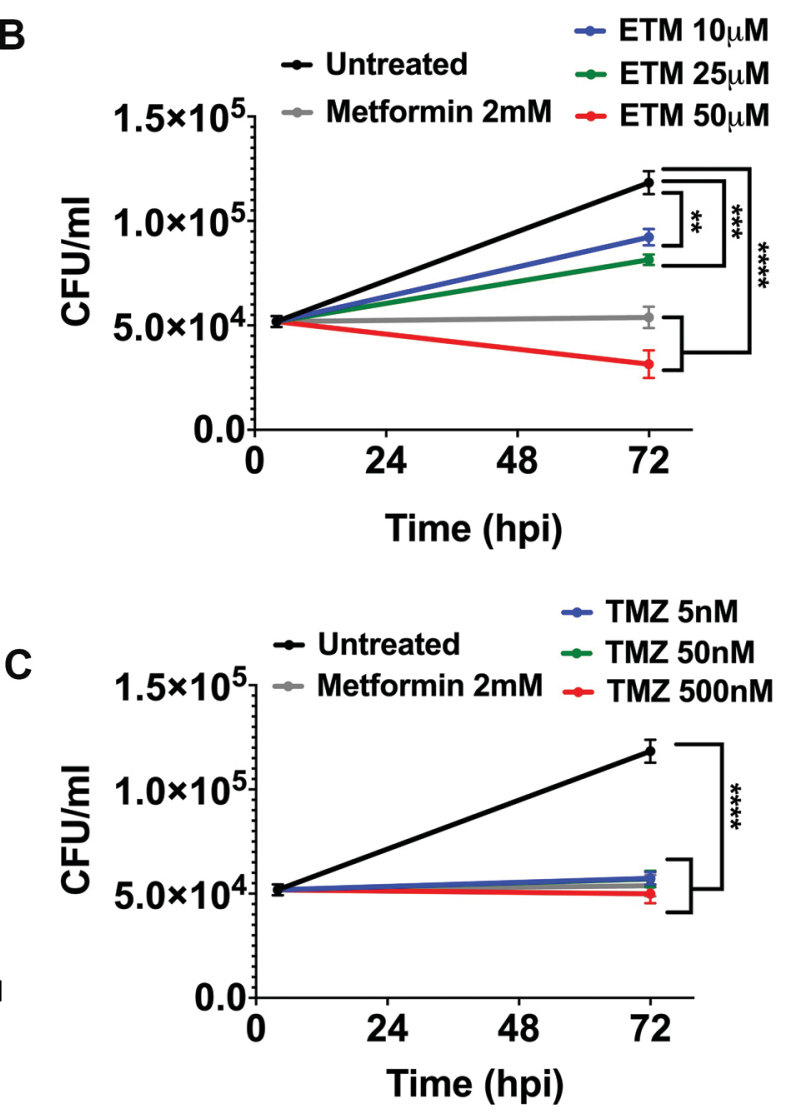

E

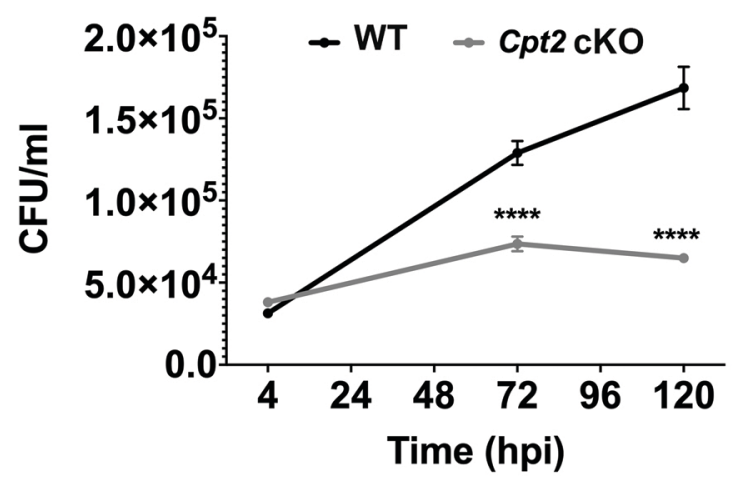

G

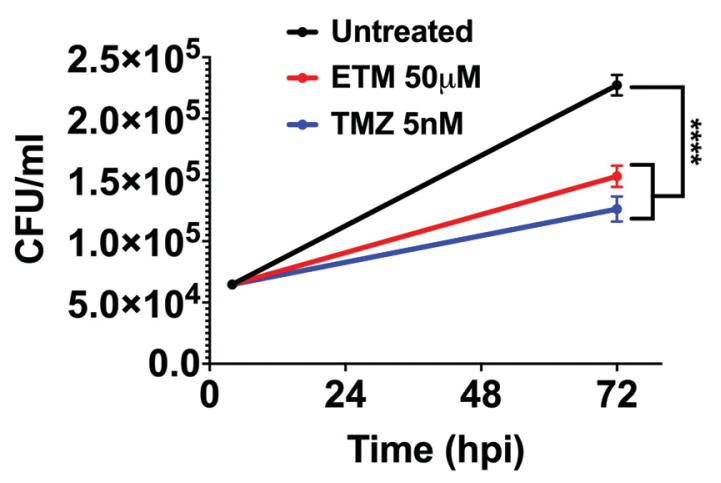


bioRxiv preprint doi: https://doi.org/10.1101/799619; this version posted October 10,2019 . The copyright holder for this preprint (which was not Figure 2
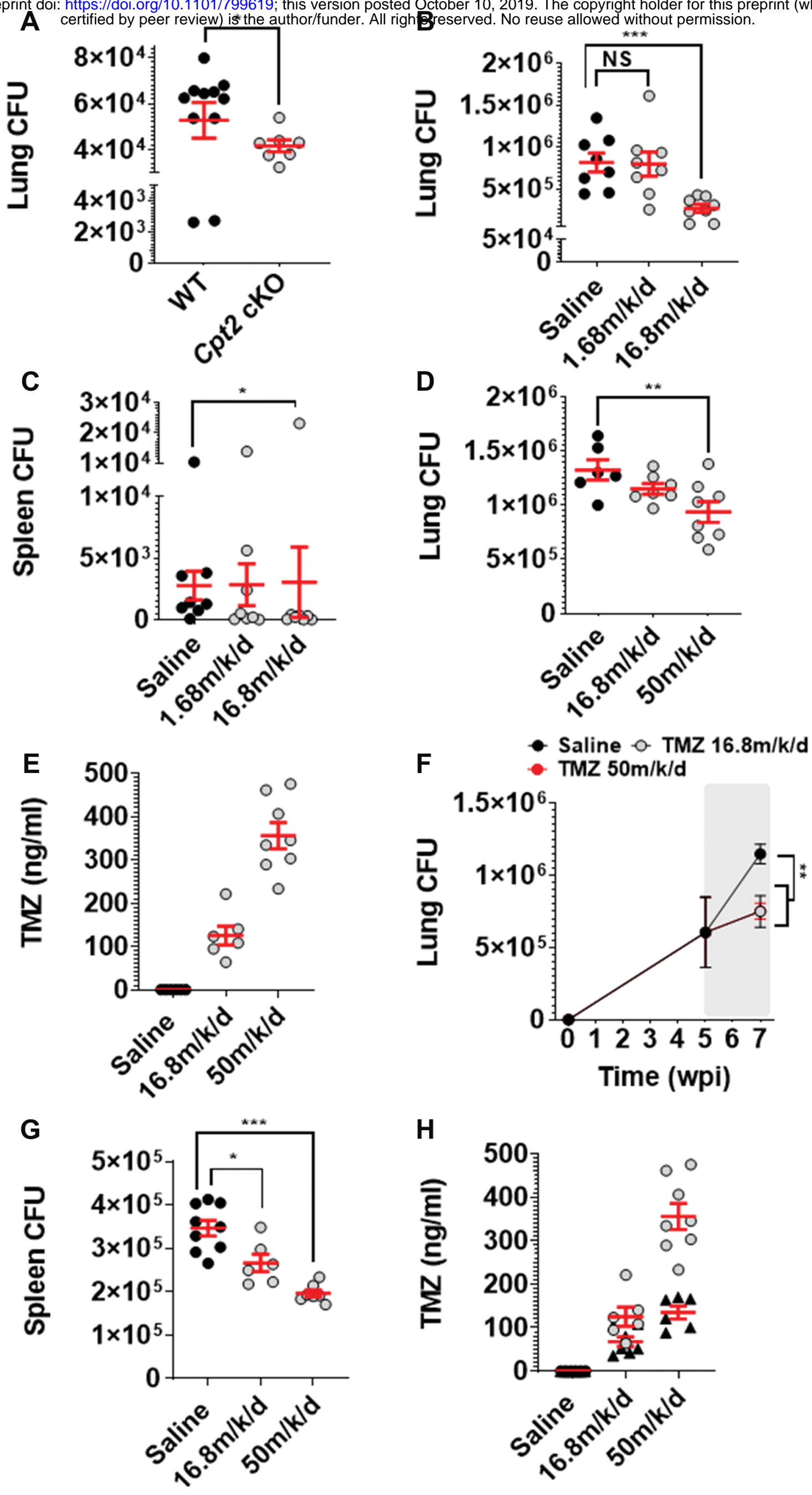

Figưfipexinpreprint doi: https://doi.org/10.1101/799619; this version posted October 10, 2019. The copyright holder for this preprint (which was not
certified by peer review) is the author/funder. All rights reserved. No reuse allowed without permission.

A

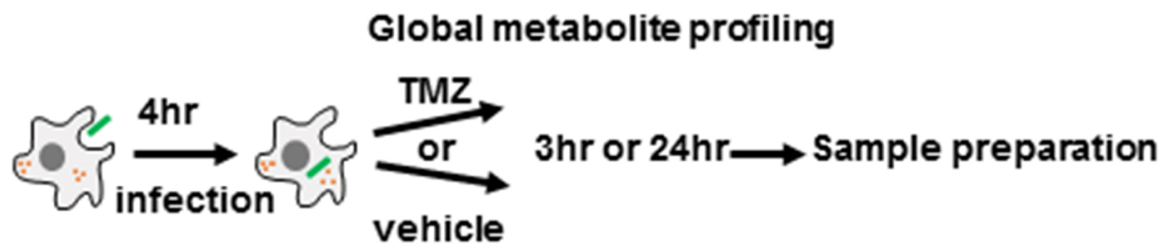

B

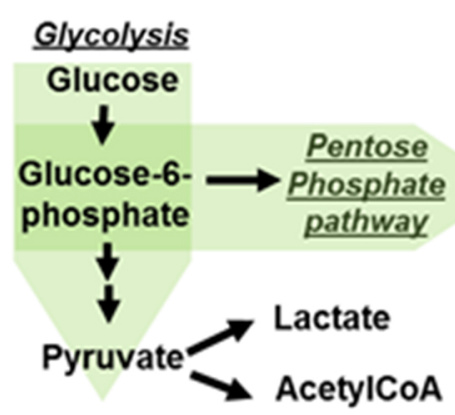

\begin{tabular}{|c|c|c|c|c|c|}
\hline \multirow{3}{*}{ Sub Pathway } & \multirow{3}{*}{ Biochemical Name } & \multicolumn{4}{|c|}{$\frac{\mathrm{TB}}{\mathrm{Ctrl}}$} \\
\hline & & \multicolumn{2}{|c|}{ None } & \multicolumn{2}{|c|}{ Drug } \\
\hline & & $3 \mathrm{H}$ & $24 \mathrm{H}$ & $3 \mathrm{H}$ & $24 \mathrm{H}$ \\
\hline \multirow{7}{*}{$\begin{array}{c}\text { Glycolysis, } \\
\text { Gluconeogene } \\
\text { sis, and } \\
\text { Pyruvate } \\
\text { Metabolism }\end{array}$} & glucose & 0.83 & 1.02 & 0.83 & 0.92 \\
\hline & glucose 6-phosphate & 1.55 & 1.12 & 1.50 & 1.52 \\
\hline & $\begin{array}{l}\text { fructose 1,6-diphosphate/glucose } \\
1,6 \text {-diphosphate/myo-inositol } \\
\text { diphosphates }\end{array}$ & 1.32 & 1.55 & 1.34 & 1.81 \\
\hline & 3-phosphoglycerate & 1.20 & 1.20 & 1.20 & 1.39 \\
\hline & phosphoenolpyruvate (PEP) & 0.97 & 1.27 & 1.10 & 1.49 \\
\hline & pyruvate & 0.91 & 1.29 & 1.12 & 1.28 \\
\hline & lactate & 1.14 & 1.48 & 1.33 & 1.48 \\
\hline \multirow{2}{*}{$\begin{array}{l}\text { Pentose } \\
\text { Phosphate } \\
\text { Pathway }\end{array}$} & $\begin{array}{l}\text { 5-phosphoribosyl diphosphate } \\
\text { (PRPP) }\end{array}$ & 1.03 & 1.65 & 1.13 & 1.96 \\
\hline & sedoheptulose-7-phosphate & 1.26 & 1.43 & 1.27 & 1.79 \\
\hline
\end{tabular}

C
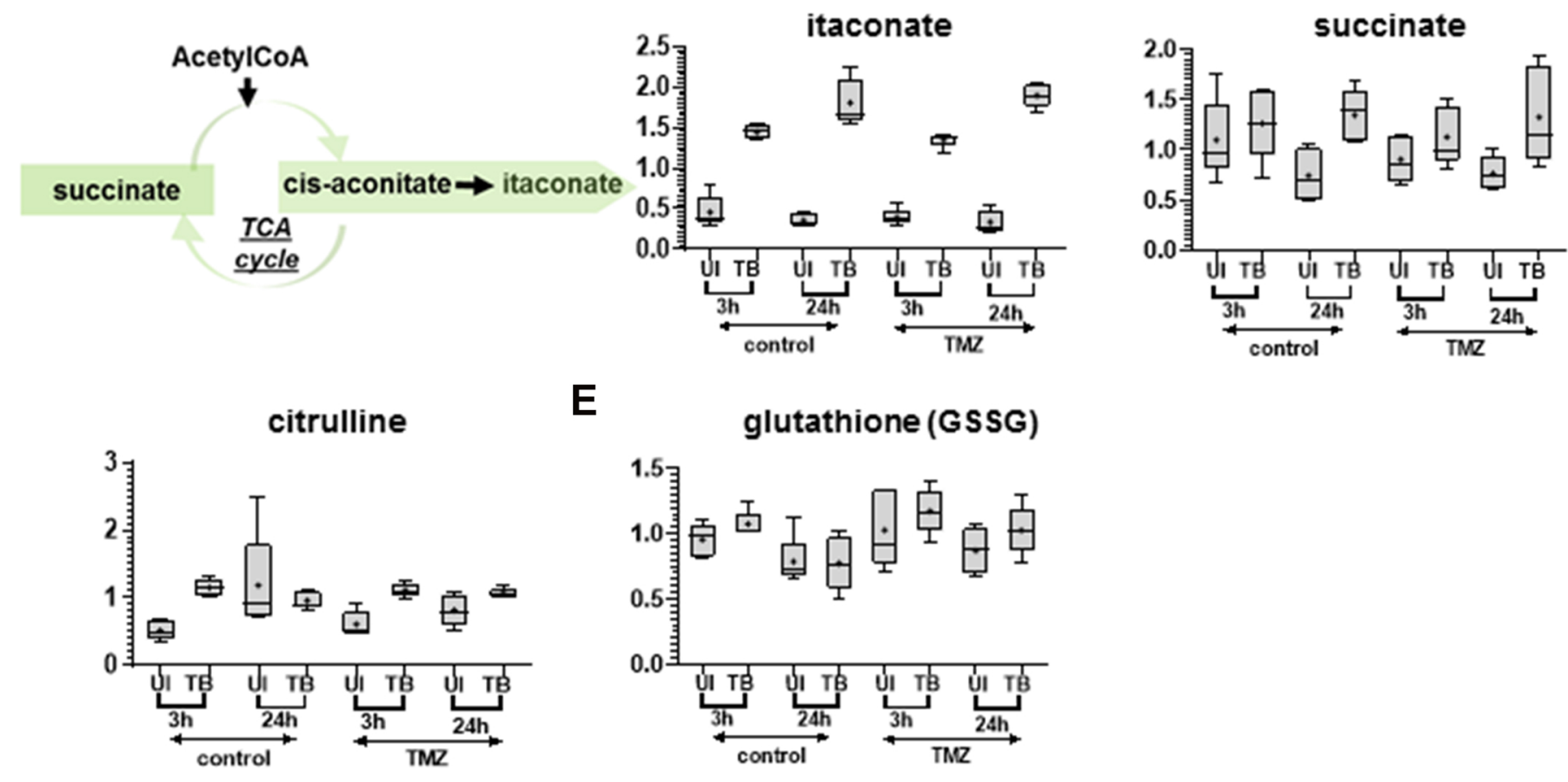

D
E glutathione (GSSG)

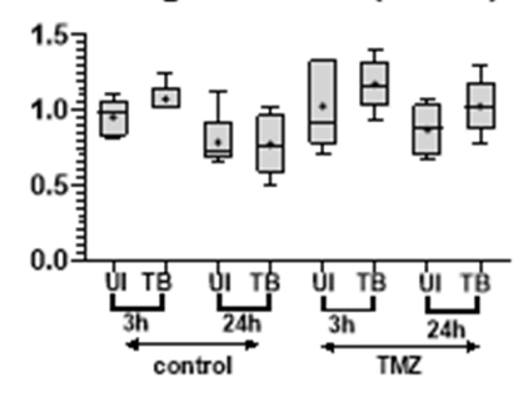


Figure 4

A

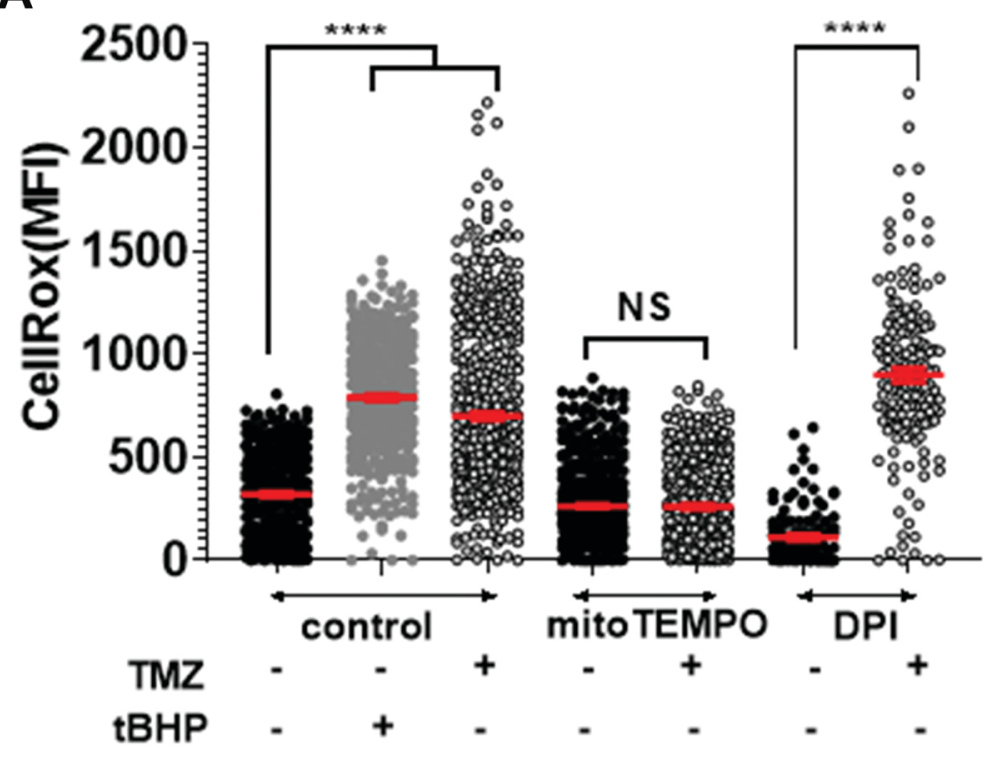

B

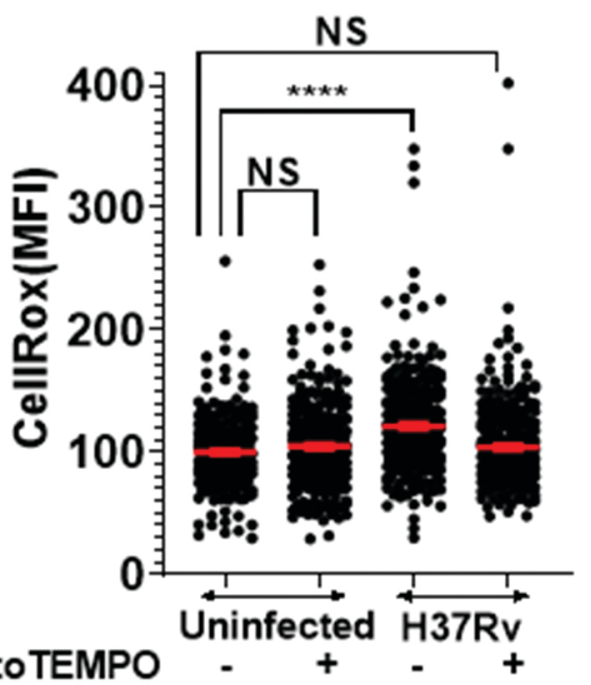

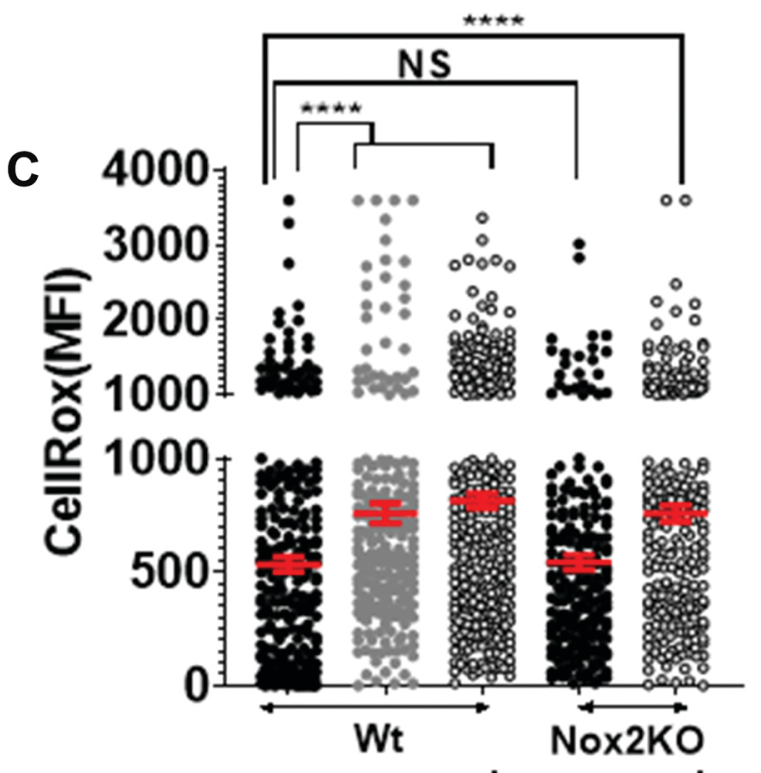

$\mathrm{TMZ}$ - - + - +
D

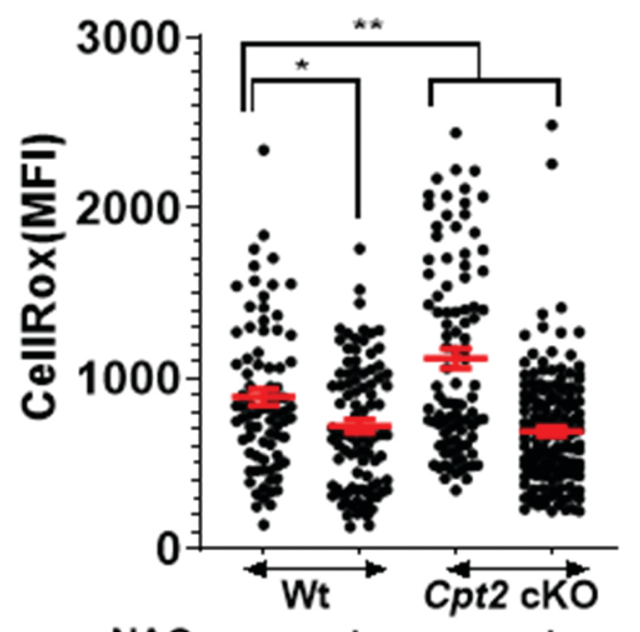

F

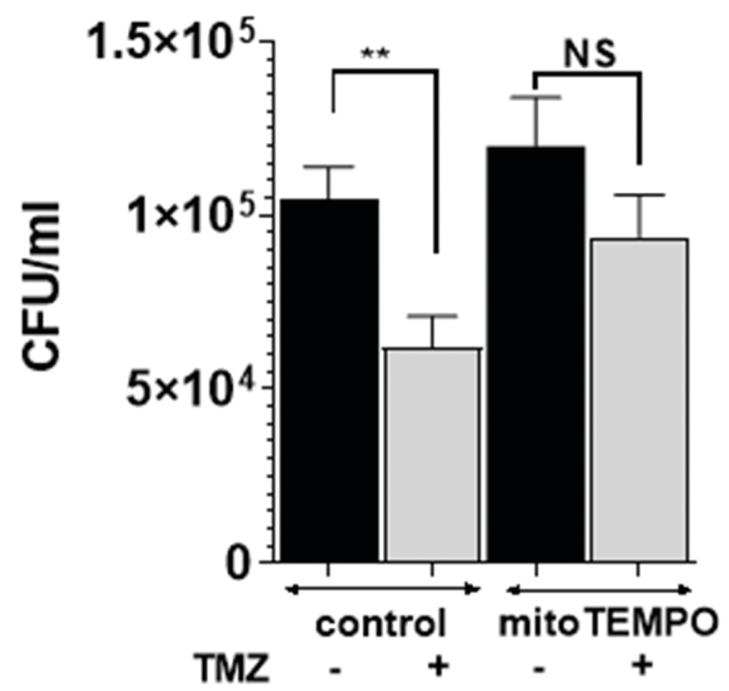

Rotenone 
Figure 5

A
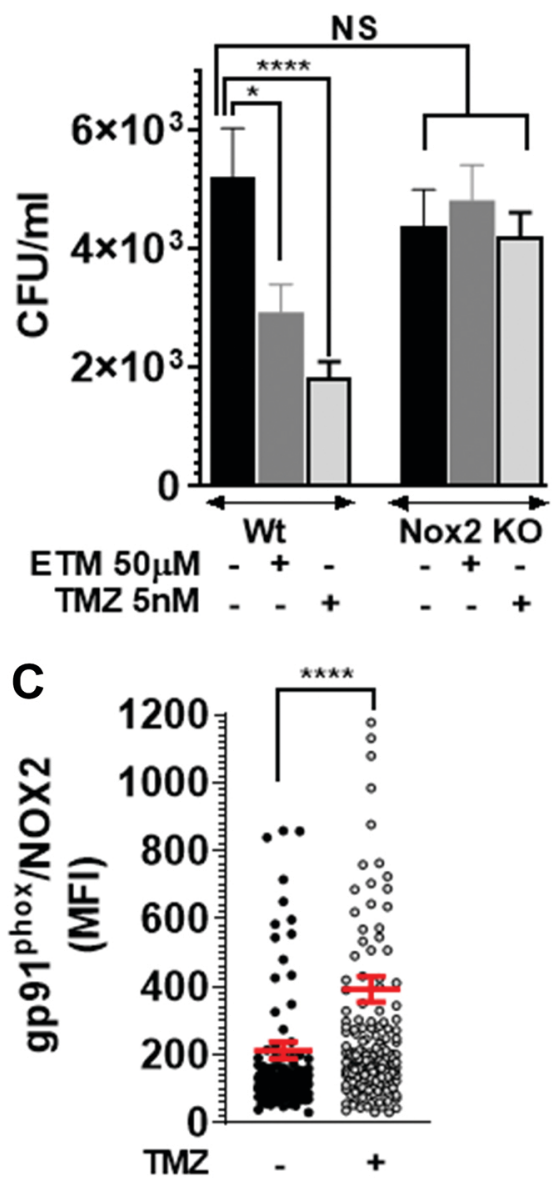
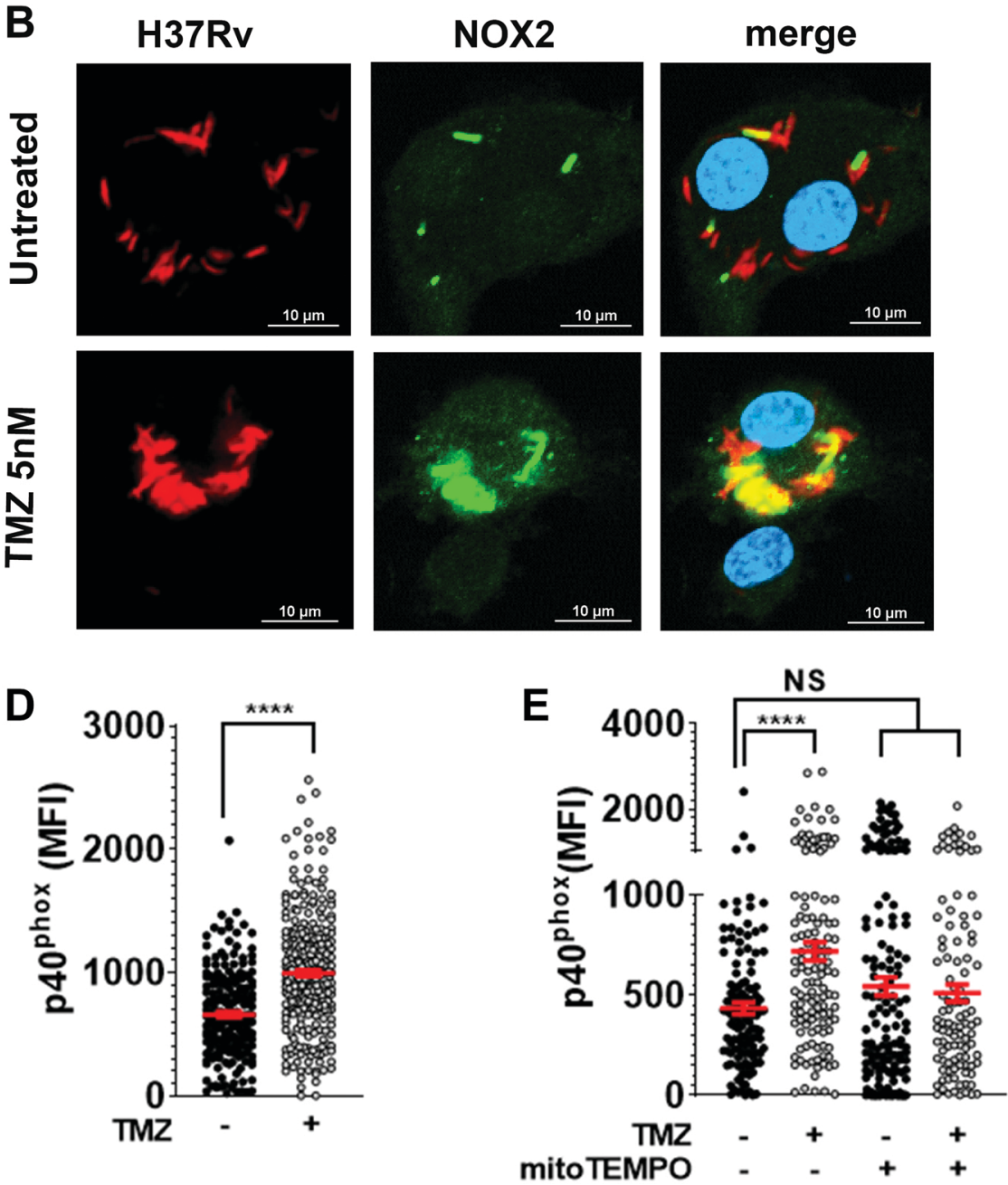
Figure 6

bioRxiv preprint doi: https://doi.org/10.1101/799619; this version posted October 10, 2019. The copyright holder for this preprint (which was not

A

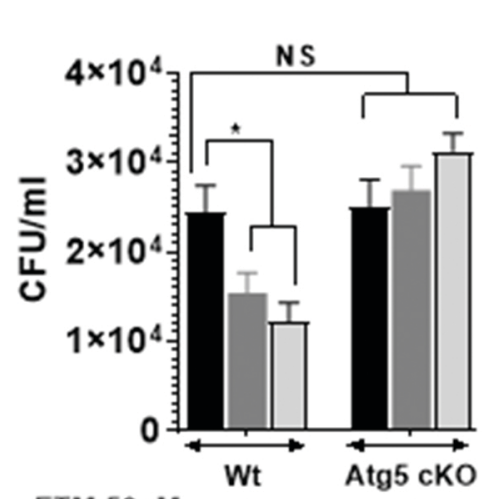

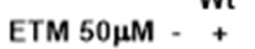

$\mathrm{TMZ} 5 \mathrm{nM}$ - - +
B

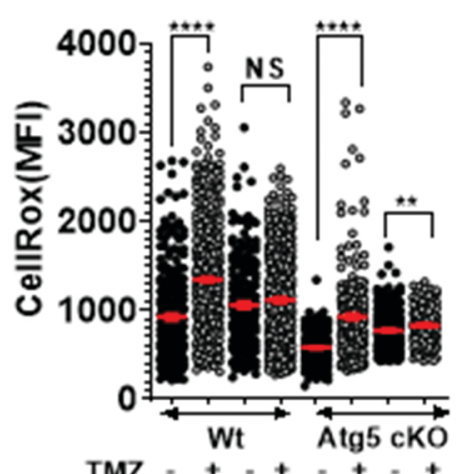

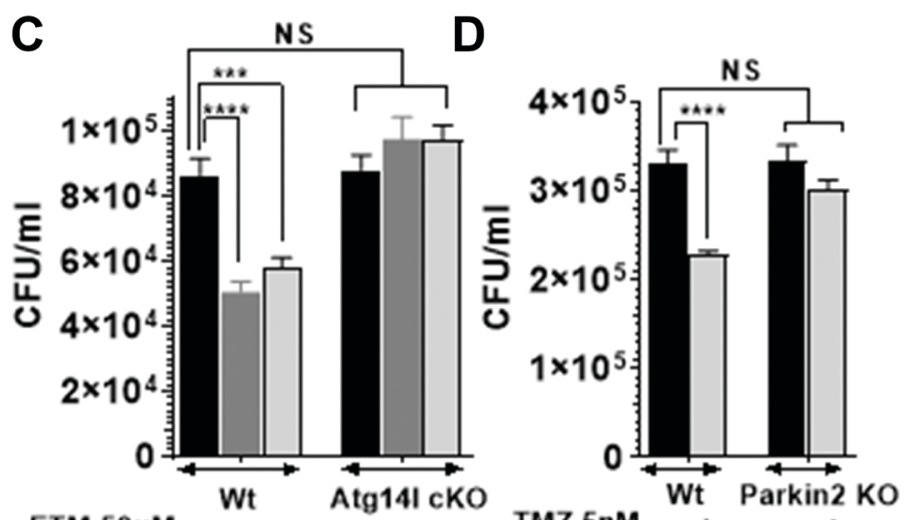

ETM $50 \mu \mathrm{M}-+\ldots$
E

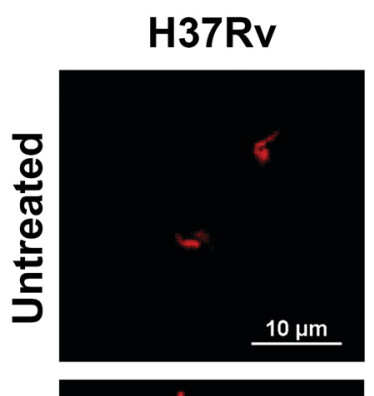

S
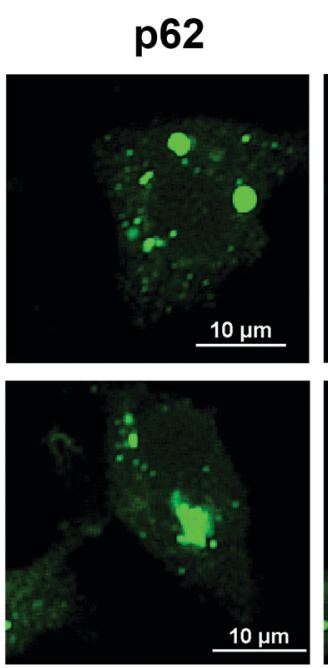

H

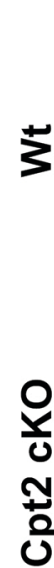
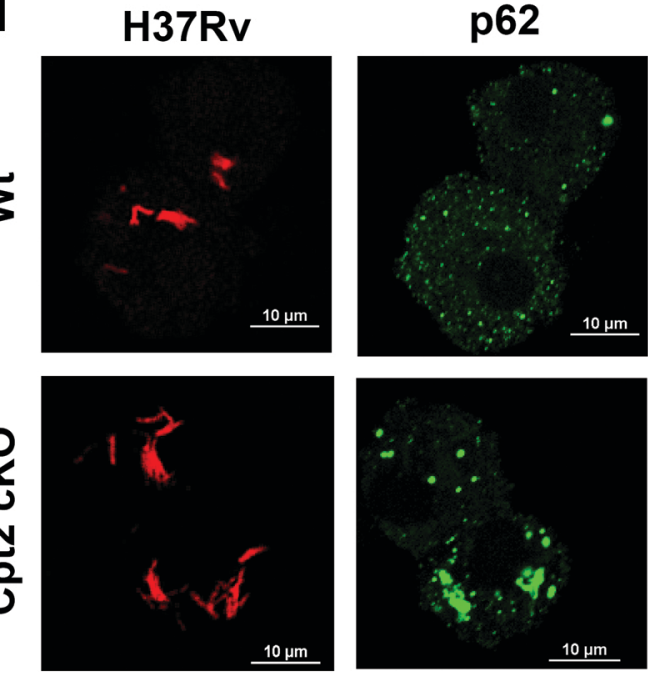

merge
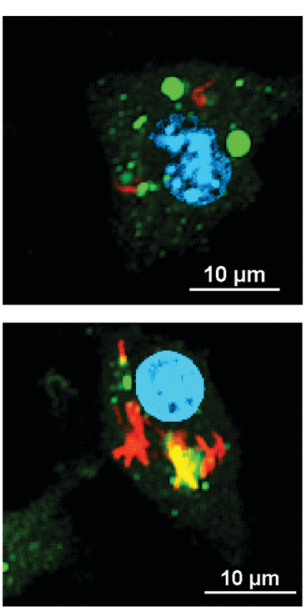

F
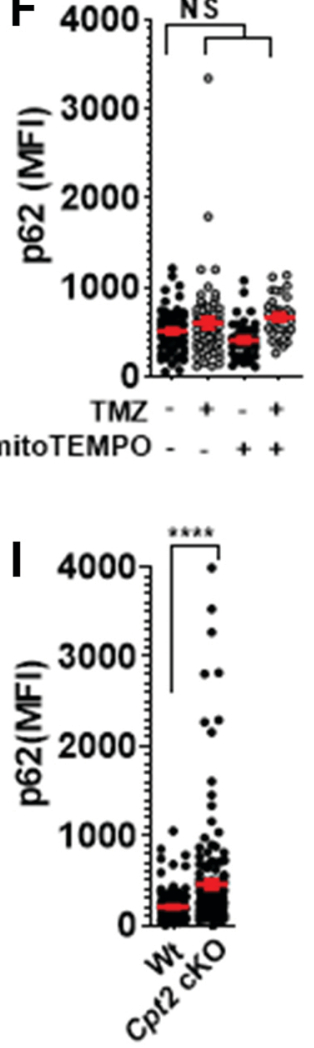
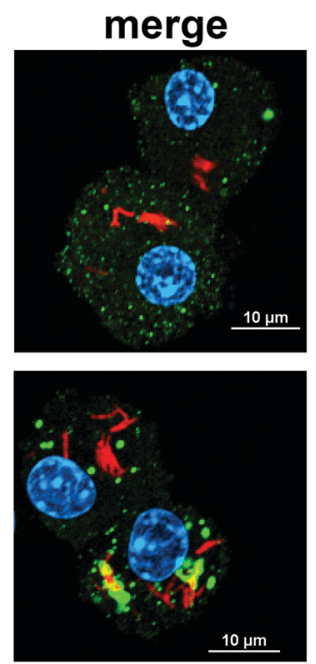
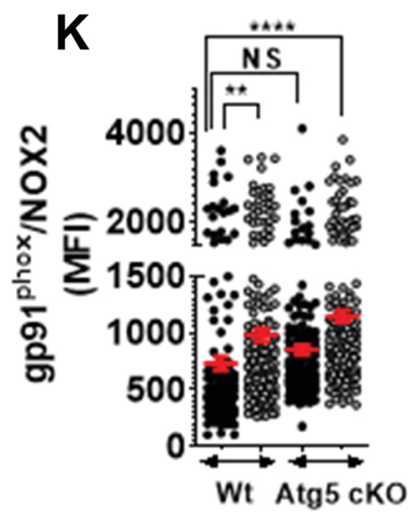

TMZ 5nM - + - +

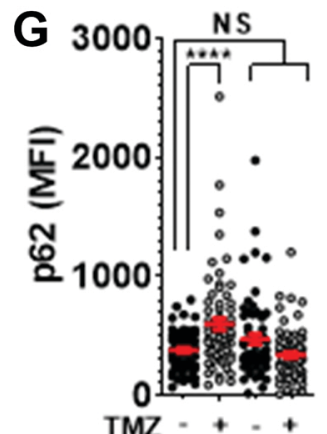

mitoTEMPO - - ++

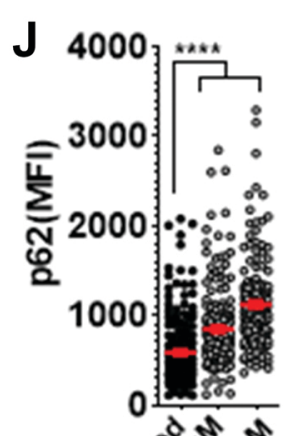

$\mathbf{L}$

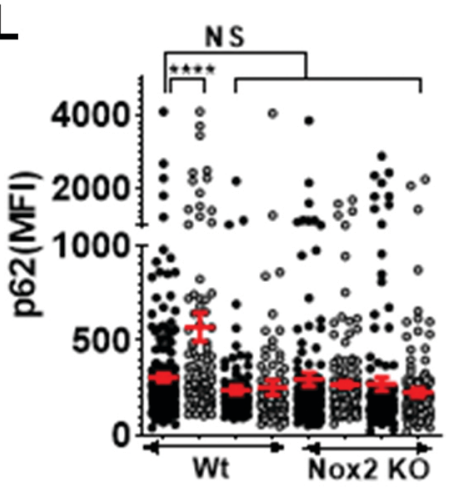

$\mathrm{TMZ}++\cdots+\cdots+\cdots+$

mitoTEMPO $-t_{+}+t_{+}+$ 


\section{SUPPLEMENTAL INFORMATION}

785

Figure S1. Inhibition of macrophage FAO restricts intracellular M. tuberculosis growth.

(A) Murine BMDMs were infected with live-dead reporter expressing H37Rv and were either untreated or treated with $50 \mathrm{nM} \mathrm{TMZ}$ for 6 days. The reporter Mtb strain constitutively expresses mCherry and has GFP controlled by a tetracycline-inducible promoter. To induce GFP expression, $200 \mathrm{nM}$ anhydrotetracycline was added 2 days after infection. Metabolically active bacteria express both GFP and mCherry, whereas dead bacteria are only mCherry-positive. Representative images show more dead Mtb upon TMZ treatment as compared to control. Scale bar $=10 \mu \mathrm{m}$. (B) Macrophage were uninfected or infected with Mtb for $72 \mathrm{~h}$, and cell viability was assessed using calcein dye mean fluorescence intensity (MFI). Plot shows cell viability expressed as \% of uninfected. Data shows mean $+/$ - s.e.m., ${ }^{*} \mathrm{P}=0.03,{ }^{*} \mathrm{P} \leq 0.0005$ ordinary one-way ANOVA.

H37Rv expressing the Vibrio harvei luciferase (Rv-lux) was cultured in 7H9 broth with or without $1 \mathrm{mM}$ TMZ for 7 days. Plot shows bacterial growth as assessed by relative luminescence units (RLU). (D) Minimal inhibitory concentration (MIC) of etomoxir, oxfenicine, metformin, and isoniazid (INH, positive control) was determined by culturing H37Rv for 4 days in $7 \mathrm{H} 9$ broth supplemented with various doses of inhibitors and measuring absorbance at $600 \mathrm{~nm}$. (E) Intracellular growth of M. abscessus in BMDMs that were untreated or treated with indicated concentrations of amikacin or TMZ for 48 hours. Data shows mean $+/-$ s.e.m., ${ }^{* * * *} \mathrm{P} \leq 0.0001$ ordinary one-way ANOVA. (F) BODIPY fluorescent dye was used to stain lipid bodies in BMDMs that were infected for 24 hours with Mtb and either untreated or treated with TMZ. Shown here is mean $+/$ - s.e.m of the $\%$ area in a cell occupied by BODIPY stain. $* * * * \mathrm{P} \leq 0.0001$, unpaired Student's t-test with Welch's correction. Using extracellular flux analysis, we quantified the (G) oxygen consumption rate (OCR) in uninfected control and Cpt2 cKO BMDMs treated with 5nM 
807 TMZ or solvent control for $3 \mathrm{~h}$, followed by sequential addition of oligomycin (A), FCCP (B), and 808 rotenone plus antimycin (C). Data shows average +/- s.d. of 16 replicates.

810 Figure S2. Pharmacokinetics study of TMZ in mice.

811 The half-life of TMZ was determined in C57BL/6 mice that were administered the inhibitor (A)

812 orally at $15 \mathrm{mg} / \mathrm{kg}$ or (B) intravenously at $3 \mathrm{mg} / \mathrm{kg}$. (C) TMZ (10.66 mg/kg/day) was administered

813 to female $\mathrm{C} 57 \mathrm{Bl} / 6$ mice $(\mathrm{n}=5)$ for 48 hours using Alzet osmotic pumps and achieved $\mathrm{C}_{\text {ave- }} 34.5$

$814 \mathrm{ng} / \mathrm{ml}$. (D) Protein binding of TMZ in mouse plasma (C57BL/6) was measured using rapid 815 equilibrium dialysis $(\mathrm{n}=3)$. Warfarin was used as a control. In humans TMZ is reportedly weakly 816 protein bound $(\sim 16 \%)$ (Harpey et al., 1988$)$.

Figure S3. FAO inhibition enhances metabolic changes in response to Mtb infection.

819 Unbiased metabolite profiling was performed in BMDMs to characterize the effect of Mtb

820 infection and TMZ treatment. (A) Macrophage response to infection involved perturbations in

821 redox homeostasis. Increased glutathione synthesis was suggested by increased methionine to

822 cysteine conversion pathway metabolites SAM, SAH and cystathione. Ophthalmate, a

823 compositional derivative of glutathione, can reflect enhanced activity of glutathione synthetase.

824 Marked elevations were observed in gamma-glutamyl amino acids, which are formed when

825 gamma-glutamyl transpeptidase transfers the gamma-glutamyl moiety of glutathione to acceptor

826 amino acids. Infection significantly increased dihydrobiopterin and biopterin. These metabolites

827 are oxidized forms of tetrahydrobiopterin, which is a cofactor for all NOS isoforms and its

828 depletion is associated with enzyme uncoupling and ROS generation. (B) Increases in membrane 
829 phospholipids and sphingomyelin were detected 24 hpi, which were enhanced in presence of TMZ.

830 (C) Tryptophan metabolism was increased with infection 24 hpi. Elevated levels of serotonin were

831 observed, with more pronounced accumulation upon TMZ treatment. Graph shows ScaledImpData

832 of serotonin in uninfected (UI) and infected (TB) samples at the indicated time points. The line

833 and dot show median and mean, respectively. Tryptophan can be converted by indoleamine 2,3-

834 dioxygenase (IDO) to kynurenine, which is further utilized for nicotinamide metabolism. TMZ

835 treatment in infected cells increased levels of metabolites in the nicotinamide pathway, suggesting

836 it might improve infection control in combination with SIRT 1 activators, as sirtuins are NAD+-

837 dependent class III histone deacetylases and activating sirtuin 1 (SIRT 1) is protective in Mtb

838 infection (Cheng et al., 2017). Other metabolic changes during infection included (D) increase in

839 nucleotide sugars, with a marked increase in UDP-N-acetylglucosamine/galactosamine (UDP-

840 GlcNAc) when FAO was inhibited. These metabolites are utilized in protein and lipid

841 glycosylation reactions, and UDP-GlcNAc biosynthesis was shown to be important for M2

842 macrophage polarization (Jha et al., 2015). (E) TMZ-induced accumulation in uracil-containing

843 pyrimidines in Mtb infected macrophages. This may indicate an increase in dihydroorotate

844 dehydrogenase (DHODH) activity, which catalyzes a rate-limiting step in the pyrimidine pathway.

845 Since DHODH can supply electrons downstream of complex I of the mitochondrial ETC, it can

846 potentially contribute to RET-ROS production. (A-F) Heatmaps show metabolite ratios in Mtb

847 infected and uninfected (TB/Ctrl) macrophages, that were untreated (None) or TMZ treated (Drug)

848 for 3 or 24 h. Here, significant difference $(p \leq 0.05$, three-way ANOVA) between groups are colored

849 in green for metabolite ratio of $<1$ and red for ratio of $>1$. Light green and red colors show groups

850 that missed the statistical cutoff for significance $0.05<\mathrm{p}<0.10$.) 
852 Figure S4. Pro-inflammatory cytokine secretion in macrophages treated with TMZ.

853 Supernatants from uninfected or Mtb-infected BMDMs that were untreated or treated with

854 indicated concentrations of TMZ were harvested 24 and 72 hpi. The levels of pro-inflammatory

855 cytokines and chemokines (A) TNF- $\alpha$ (B) IL-6, (C) CXCL2, (D) IFN- $\beta$, (E) CCL2, and (F)

856 CXCL10 were measured using a Milliplex MAP Mouse Cytokine/Chemokine Magnetic Bead

857 Panel. $* \mathrm{P} \leq 0.02, * * \mathrm{P}=0.004$ calculated using ordinary one-way ANOVA.

858

859 Table S1. Metabolite Differences Associated with Mtb infection and TMZ treatment

860

861

862

863

864

865

866

867

868

869

870

871 
Figure S1

A

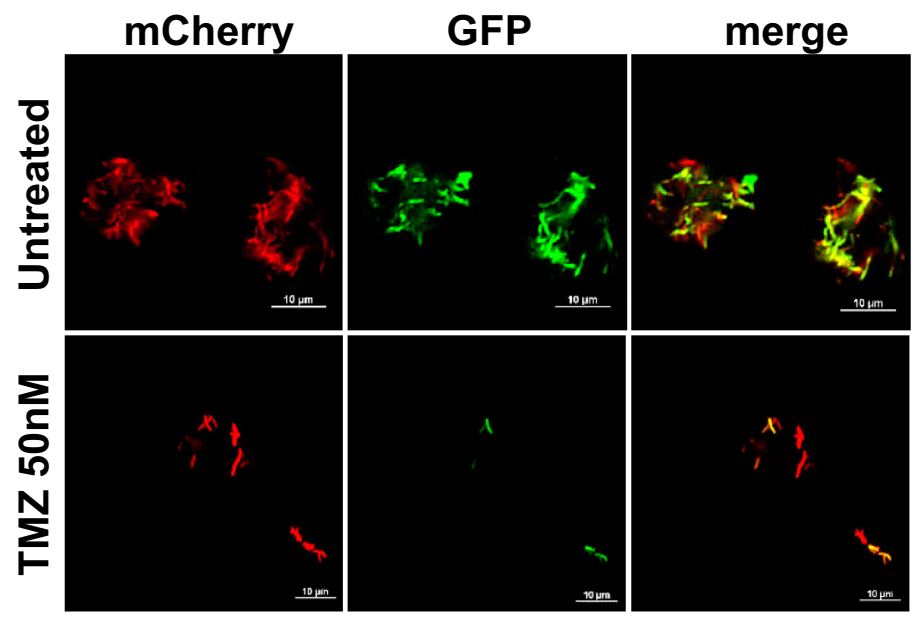

c

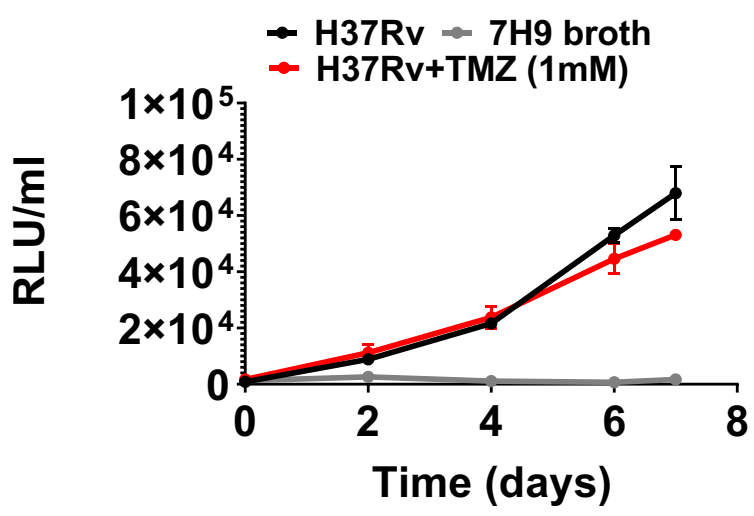

E

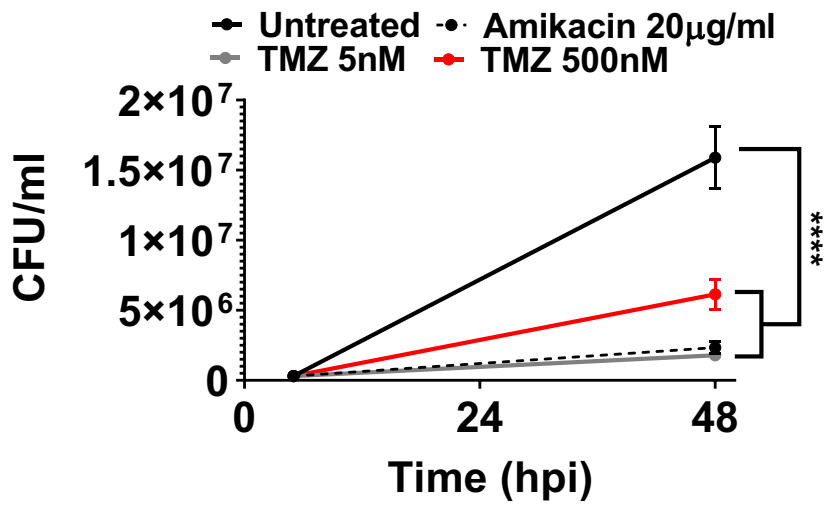

G

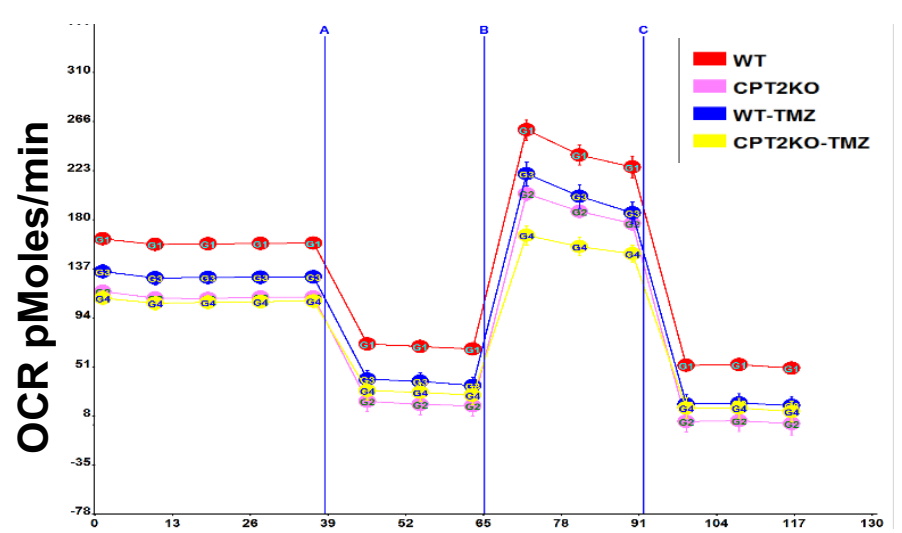

B

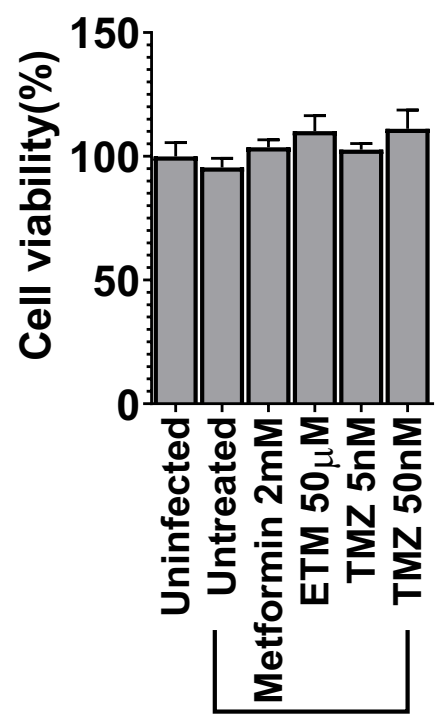

H37Rv

D

\begin{tabular}{c|c}
$\begin{array}{c}\text { Antitubercular activity against } \\
\text { H37Rv in liquid media }\end{array}$ \\
\hline Drug & MIC \\
\hline Etomoxir & $>250 \mu \mathrm{M}$ \\
\hline Oxfenicine & $>140 \mathrm{mM}$ \\
\hline Metformin & $>15 \mathrm{mM}$ \\
\hline INH & $<0.3 \mu \mathrm{M}$ \\
\hline
\end{tabular}

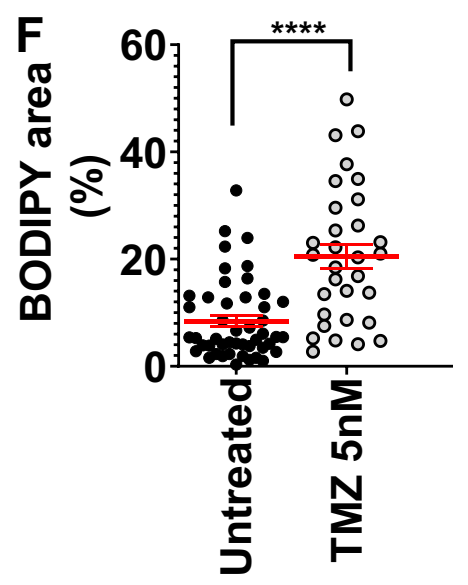




\section{Figure S2}

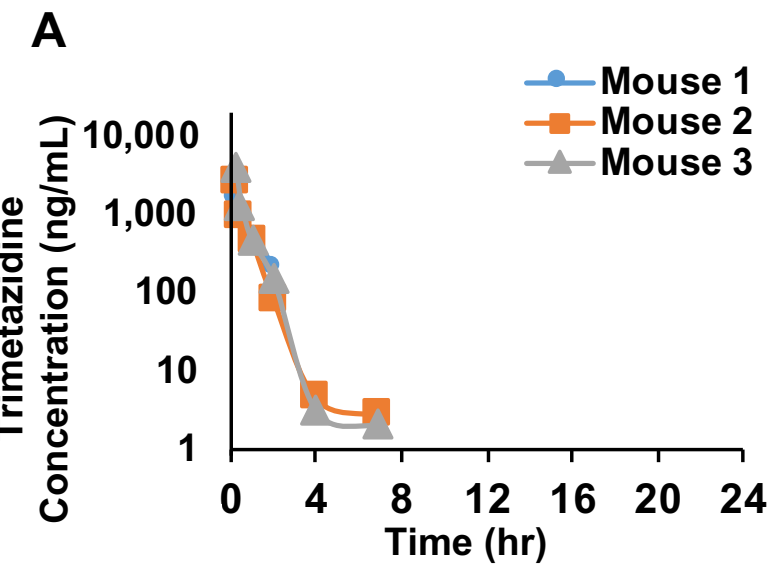

C

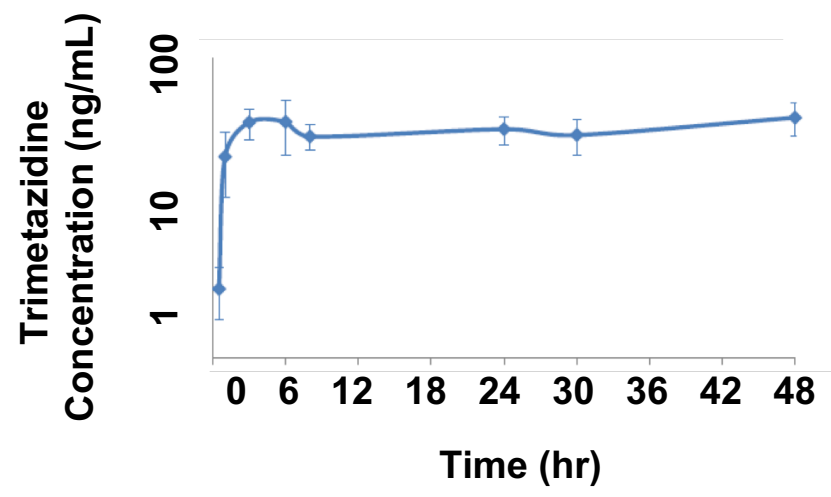

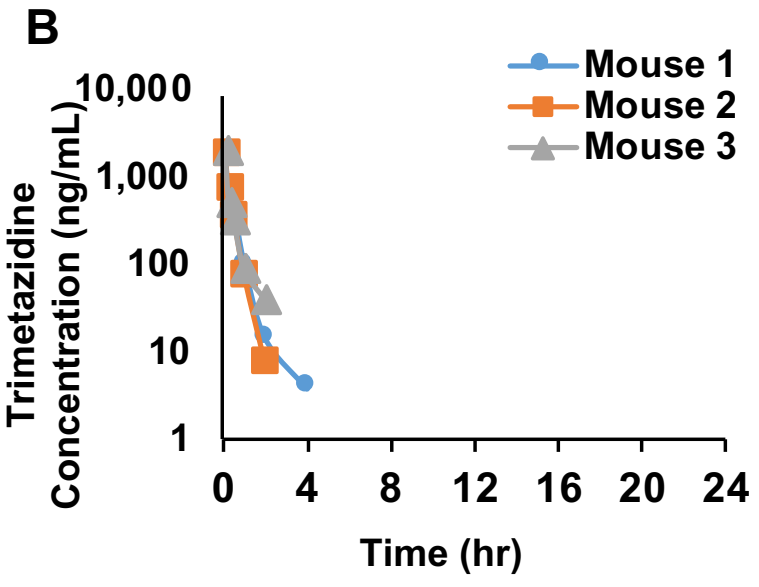

D

\begin{tabular}{|c|c|c|}
\hline \multicolumn{2}{|c|}{ Protein Binding in Mouse Plasma } \\
\hline Compound & $\begin{array}{c}\text { Percent } \\
\text { Free } \\
\text { Fraction }\end{array}$ & $\begin{array}{c}\text { Percent } \\
\text { Recovery }\end{array}$ \\
\hline Trimetazidine & 64.86 & 97.94 \\
\hline Warfarin & 4.55 & 110.55 \\
\hline
\end{tabular}




\section{A Redox homeostasis}

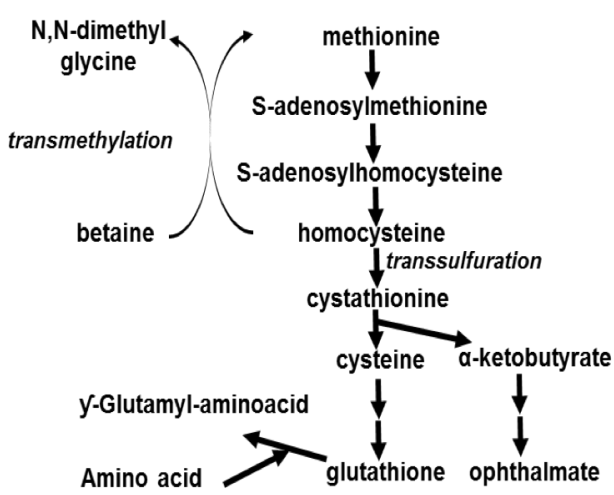

\begin{tabular}{|c|c|c|c|c|c|}
\hline \multirow{4}{*}{ Sub Pathway } & \multirow{4}{*}{ Biochemical Name } & \multirow{2}{*}{\multicolumn{4}{|c|}{$\frac{\text { TB }}{\text { Ctrl }}$}} \\
\hline & & & & & \\
\hline & & \multicolumn{2}{|c|}{ None } & \multicolumn{2}{|c|}{ Drug } \\
\hline & & $3 \mathrm{H}$ & $24 \mathrm{H}$ & $3 \mathrm{H}$ & $24 \mathrm{H}$ \\
\hline \multirow{3}{*}{ Methionine and cysteine metabolism } & S-adenosylmethionine (SAM) & 1.01 & 1.11 & 1.07 & 1.41 \\
\hline & S-adenosylhomocysteine (SAH) & 0.90 & 1.30 & 1.02 & 1.48 \\
\hline & cystathionine & 0.98 & 1.34 & 1.30 & 1.75 \\
\hline Glutathione metabolism & ophthalmate & 1.68 & 0.98 & 1.49 & 1.23 \\
\hline \multirow{11}{*}{ Gamma-glutamyl amino acid } & gamma-glutamylcysteine & 1.41 & 0.98 & 1.39 & 0.97 \\
\hline & gamma-glutamylglutamate & 1.46 & 1.47 & 1.50 & 1.46 \\
\hline & gamma-glutamylglutamine & 2.19 & 1.93 & 2.14 & 2.16 \\
\hline & gamma-glutamylglycine & 1.63 & 0.80 & 1.46 & 1.07 \\
\hline & gamma-glutamylisoleucine* & 1.53 & 1.00 & 1.42 & 1.15 \\
\hline & gamma-glutamylleucine & 1.36 & 0.82 & 1.29 & 1.04 \\
\hline & gamma-glutamyl-epsilon-lysine & 1.25 & 0.72 & 1.35 & 0.64 \\
\hline & gamma-glutamylmethionine & 1.71 & 2.28 & 1.45 & 2.40 \\
\hline & gamma-glutamylphenylalanine & 2.73 & 1.84 & 2.36 & 2.34 \\
\hline & gamma-glutamylthreonine & 1.55 & 0.86 & 1.35 & 0.90 \\
\hline & gamma-glutamylvaline & 1.41 & 0.83 & 1.29 & 0.99 \\
\hline Tetrahydrobiopterin Metabolism & dihydrobiopterin & 1.46 & 3.44 & 1.24 & 3.92 \\
\hline Pterin Metabolism & pterin & 1.42 & 3.16 & 1.69 & 3.38 \\
\hline
\end{tabular}

\section{B}

\section{Changes in Phospholipids and sphingolipids}

\begin{tabular}{|c|c|c|c|c|c|}
\hline \multirow{4}{*}{ Sub Pathway } & \multirow{4}{*}{ Biochemical Name } & \multirow{2}{*}{\multicolumn{4}{|c|}{ TB }} \\
\hline & & & & & \\
\hline & & \multicolumn{2}{|c|}{ None } & \multicolumn{2}{|c|}{ Drug } \\
\hline & & $3 \mathrm{H}$ & $24 \mathrm{H}$ & $3 \mathbf{H}$ & $24 \mathrm{H}$ \\
\hline \multirow{9}{*}{$\begin{array}{l}\text { Phosphatidylcholine } \\
\text { (PC) }\end{array}$} & 1-myristoyl-2-palmitoyl-GPC (14:0/16:0) & 1.04 & 1.27 & 0.99 & 1.41 \\
\hline & 1-myristoyl-2-arachidonoyl-GPC (14:0/20:4)* & 1.15 & 1.11 & 1.12 & 1.32 \\
\hline & 1-palmitoyl-2-palmitoleoyl-GPC (16:0/16:1)* & 1.05 & 1.25 & 1.12 & 1.48 \\
\hline & 1-palmitoyl-2-linoleoyl-GPC (16:0/18:2) & 0.94 & 1.18 & 1.06 & 1.28 \\
\hline & $\begin{array}{l}\text { 1-palmitoyl-2-gamma-linolenoyl-GPC } \\
(16: 0 / 18: 3 n 6)^{*}\end{array}$ & 1.00 & 1.16 & 1.13 & 1.36 \\
\hline & $\begin{array}{l}\text { 1-palmitoyl-2-dihomo-linolenoyl-GPC } \\
(16: 0 / 20: 3 \mathrm{n} 3 \text { or } 6)^{*}\end{array}$ & 0.93 & 1.04 & 1.10 & 1.27 \\
\hline & $\begin{array}{l}\text { 1-palmitoyl-2-docosahexaenoyl-GPC } \\
(16: 0 / 22: 6)\end{array}$ & 0.95 & 1.21 & 1.05 & 1.36 \\
\hline & 1-oleoyl-2-linoleoyl-GPC (18:1/18:2)* & 1.03 & 1.19 & 1.06 & 1.19 \\
\hline & $\begin{array}{l}\text { 1-oleoyl-2-docosahexaenoyl-GPC } \\
(18: 1 / 22: 6)^{*}\end{array}$ & 0.91 & 1.13 & 1.04 & 1.34 \\
\hline \multirow{7}{*}{$\begin{array}{c}\text { Phosphatidylethanolami } \\
\text { ne (PE) }\end{array}$} & 1,2-dipalmitoyl-GPE $(16: 0 / 16: 0)^{\star}$ & 1.35 & 1.25 & 1.27 & 1.39 \\
\hline & 1-palmitoyl-2-oleoyl-GPE (16:0/18:1) & 1.05 & 1.18 & 1.11 & 1.41 \\
\hline & 1-palmitoyl-2-linoleoyl-GPE (16:0/18:2) & 1.14 & 1.42 & 1.14 & 1.79 \\
\hline & 1-palmitoyl-2-arachidonoyl-GPE (16:0/20:4)* & 1.12 & 1.26 & 1.04 & 1.55 \\
\hline & $\begin{array}{l}\text { 1-palmitoyl-2-docosahexaenoyl-GPE } \\
(16: 0 / 22: 6)^{*}\end{array}$ & 1.08 & 1.27 & 1.09 & 1.42 \\
\hline & $\begin{array}{l}\text { 1-stearoyl-2-docosahexaenoyl-GPE } \\
(18: 0 / 22: 6)^{*}\end{array}$ & 1.22 & 1.07 & 1.14 & 1.23 \\
\hline & 1-oleoyl-2-arachidonoyl-GPE (18:1/20:4)* & 1.05 & 1.14 & 1.10 & 1.38 \\
\hline Phosphatidylserine (PS) & 1-palmitoyl-2-oleoyl-GPS (16:0/18:1) & 0.97 & 1.15 & 1.14 & 1.36 \\
\hline \multirow[t]{2}{*}{$\begin{array}{l}\text { Phosphatidylglycerol } \\
\text { (PG) }\end{array}$} & 1-palmitoyl-2-oleoyl-GPG (16:0/18:1) & 0.90 & 1.29 & 1.08 & 1.53 \\
\hline & 1-oleoyl-2-linoleoyl-GPG (18:1/18:2)* & 0.88 & 1.33 & 1.10 & 1.84 \\
\hline Phosphatidylinositol (PI) & 1-palmitoyl-2-arachidonoyl-GPI $(16: 0 / 20: 4)^{*}$ & 0.96 & 1.11 & 0.98 & 1.21 \\
\hline \multirow{7}{*}{ Sphingomyelins } & sphingomyelin (d18:2/24:2) ${ }^{*}$ & 1.17 & 1.22 & 0.90 & 1.80 \\
\hline & sphingomyelin (d17:1/14:0, d16:1/15:0)* & 1.31 & 1.22 & 1.22 & 1.63 \\
\hline & sphingomyelin (d18:1/14:0, d16:1/16:0) & 1.03 & 1.23 & 1.13 & 1.40 \\
\hline & sphingomyelin (d18:2/14:0, d18:1/14:1)* & 1.02 & 1.24 & 1.20 & 1.58 \\
\hline & $\begin{array}{l}\text { sphingomyelin (d17:1/16:0, d18:1/15:0, } \\
\text { d16:1/17:0)* }\end{array}$ & 1.01 & 1.17 & 1.07 & 1.28 \\
\hline & sphingomyelin (d17:2/16:0, d18:2/15:0)* & 0.97 & 1.02 & 1.42 & 1.64 \\
\hline & sphingomyelin (d18:2/16:0, d18:1/16:1)* & 1.06 & 1.22 & 1.15 & 1.35 \\
\hline Ceramide PEs & $\begin{array}{l}\text { palmitoyl-sphingosine- } \\
\text { phosphoethanolamine (d18:1/16:0) }\end{array}$ & 1.02 & 1.16 & 1.06 & 1.28 \\
\hline
\end{tabular}

\section{Changes in nucleotide sugars}

\begin{tabular}{|l|l|c|c|c|c|}
\hline \multirow{3}{*}{ Sub Pathway } & \multicolumn{3}{|c|}{ Biochemical Name } & \multicolumn{4}{|c|}{ Ctrl } \\
\cline { 3 - 6 } & & \multicolumn{3}{|c|}{} \\
\cline { 3 - 6 } & & \multicolumn{3}{|c|}{ None } & \multicolumn{2}{c|}{ Drug } \\
\cline { 3 - 6 } & & $3 \mathrm{H}$ & $24 \mathrm{H}$ & $3 \mathrm{H}$ & $24 \mathrm{H}$ \\
\hline Nucleotide Sugar & UDP-glucose & 1.33 & 1.57 & 1.44 & 1.75 \\
\cline { 2 - 6 } & UDP-galactose & 1.22 & 1.23 & 1.25 & 1.48 \\
\cline { 2 - 6 } & UDP-glucuronate & 1.34 & 1.47 & 1.38 & 1.66 \\
\cline { 2 - 6 } & guanosine 5'-diphospho-fucose & 1.01 & 1.01 & 1.05 & 1.26 \\
\cline { 2 - 6 } & UDP-N-acetylglucosamine/galactos & 1.06 & 0.90 & 1.06 & 1.83 \\
\cline { 2 - 6 } & $\begin{array}{l}\text { cytidine 5'-monophospho-N- } \\
\text { acetylneuraminic acid }\end{array}$ & 1.14 & 0.96 & 1.06 & 1.39 \\
\hline Mannose metabolism & mannose-6-phosphate & 1.50 & 1.20 & 1.45 & 2.02 \\
\hline
\end{tabular}

\section{Iryptophan metabolism}

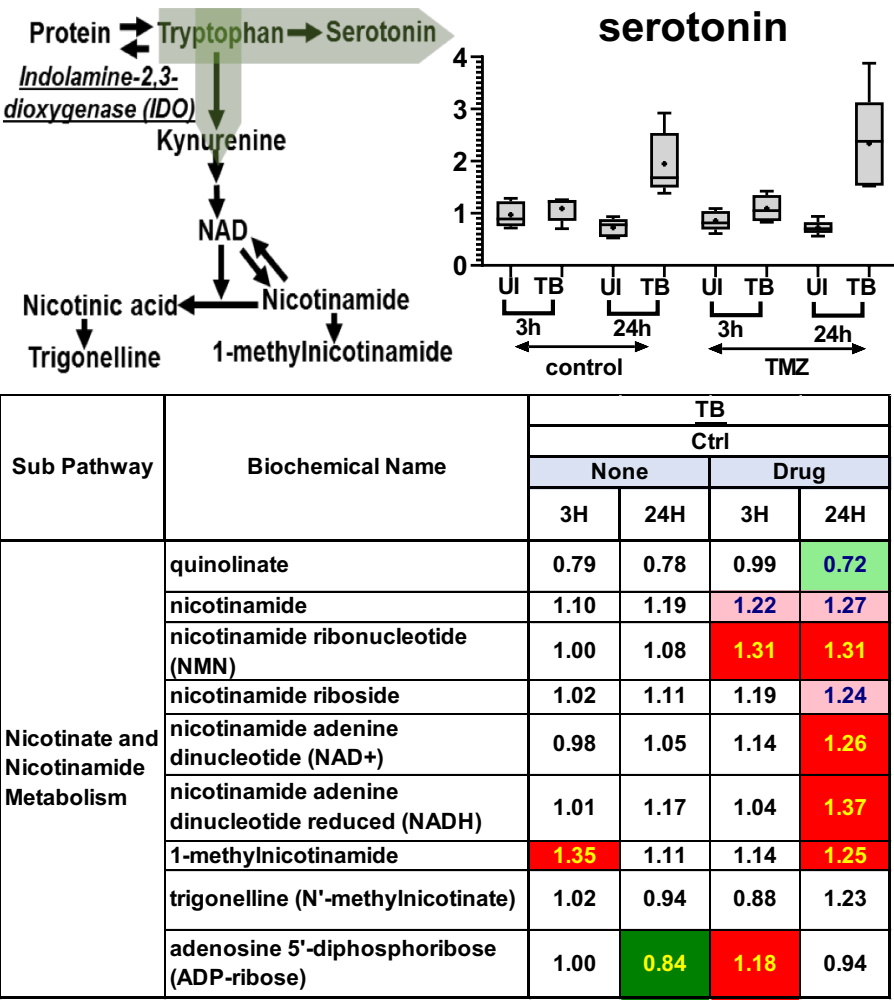

\begin{tabular}{|l|l|c|c|c|c|}
\hline Sub Pathway & \multirow{2}{*}{ Biochemical Name } & \multicolumn{4}{|c|}{ TB } \\
\cline { 3 - 6 } & & \multicolumn{4}{|c|}{ Ctrl } \\
\cline { 3 - 6 } & & \multicolumn{2}{|c|}{ None } & \multicolumn{2}{|c|}{ Drug } \\
\cline { 3 - 6 } & & $3 \mathrm{H}$ & $24 \mathrm{H}$ & $3 \mathrm{H}$ & $24 \mathrm{H}$ \\
\hline \multirow{2}{*}{$\begin{array}{l}\text { Pyrimidine } \\
\text { Metabolism, } \\
\text { Orotate containing }\end{array}$} & dihydroorotate & 1.43 & 1.82 & 1.44 & 1.66 \\
\cline { 2 - 6 } & Orotate & 1.47 & 2.26 & 1.67 & 1.43 \\
\cline { 2 - 7 } & Orotidine & 1.00 & 1.10 & 1.07 & 1.55 \\
\hline
\end{tabular}


Figure S4

\section{OUninfected \\ H37Rv \\ OH37Rv+TMZ 5nM \\ $\otimes H 37 R v+T M Z$ 50nM}

A

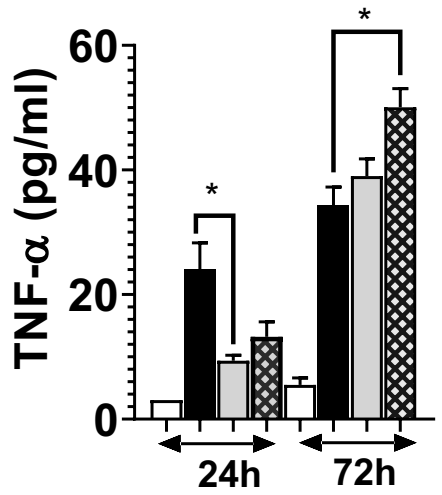

D

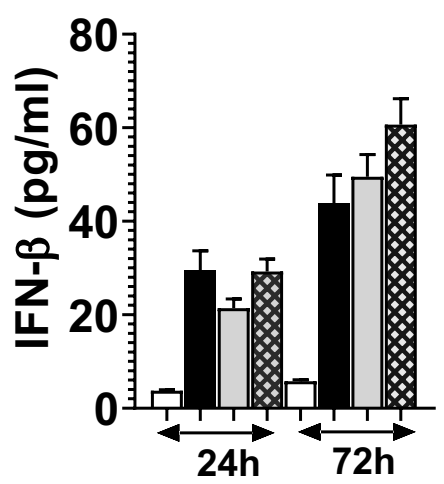

B

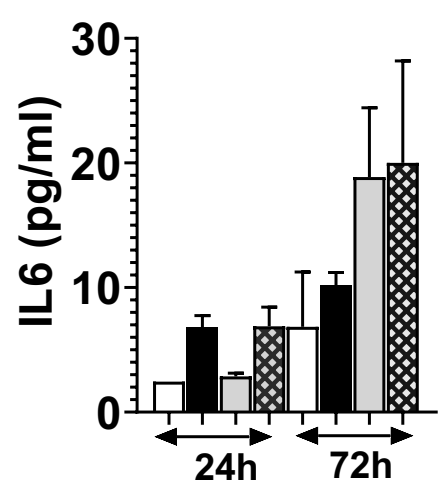

E

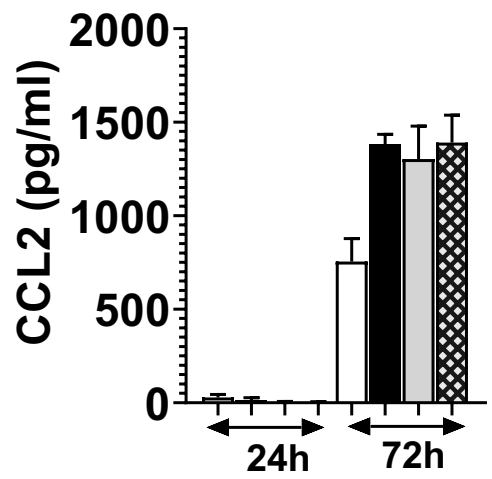

C

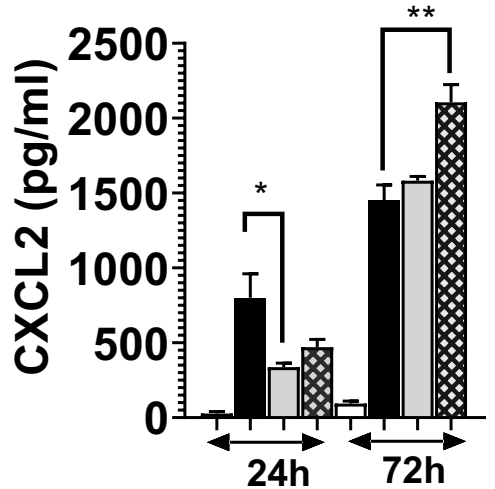

F

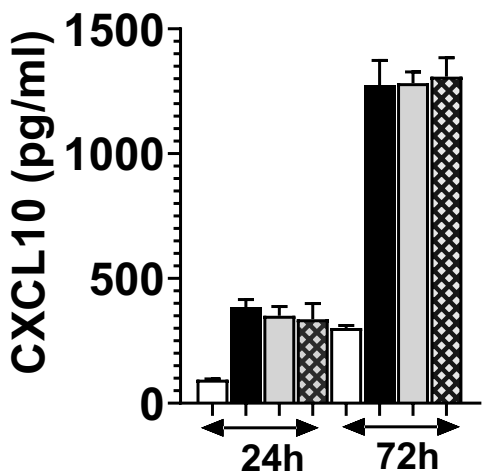




\section{REFERENCES}

873

874

875

876

877

878

879

880

881

882

883

884

885

886

887

888

Abuaita, B.H., Schultz, T.L., and O'Riordan, M.X. (2018). Mitochondria-Derived Vesicles Deliver Antimicrobial Reactive Oxygen Species to Control Phagosome-Localized Staphylococcus aureus. Cell Host Microbe 24, 625-636.e625.

Almeida, P.E., Silva, A.R., Maya-Monteiro, C.M., Töröcsik, D., D'Avila, H., Dezsö, B., Magalhães, K.G., Castro-Faria-Neto, H.C., Nagy, L., and Bozza, P.T. (2009). Mycobacterium bovis bacillus Calmette-Guérin infection induces TLR2-dependent peroxisome proliferatoractivated receptor gamma expression and activation: functions in inflammation, lipid metabolism, and pathogenesis. J Immunol 183, 1337-1345.

Arts, R.J.W., Carvalho, A., La Rocca, C., Palma, C., Rodrigues, F., Silvestre, R., Kleinnijenhuis, J., Lachmandas, E., Gonçalves, L.G., Belinha, A., et al. (2016). Immunometabolic Pathways in BCG-Induced Trained Immunity. Cell Rep 17, 2562-2571.

Bewley, M.A., Preston, J.A., Mohasin, M., Marriott, H.M., Budd, R.C., Swales, J., Collini, P., Greaves, D.R., Craig, R.W., Brightling, C.E., et al. (2017). Impaired Mitochondrial Microbicidal Responses in Chronic Obstructive Pulmonary Disease Macrophages. Am J Respir Crit Care Med $196,845-855$.

Brzostek, A., Pawelczyk, J., Rumijowska-Galewicz, A., Dziadek, B., and Dziadek, J. (2009). Mycobacterium tuberculosis is able to accumulate and utilize cholesterol. Journal of bacteriology 191, 6584-6591.

Cheng, C.Y., Gutierrez, N.M., Marzuki, M.B., Lu, X., Foreman, T.W., Paleja, B., Lee, B., Balachander, A., Chen, J., Tsenova, L., et al. (2017). Host sirtuin 1 regulates mycobacterial immunopathogenesis and represents a therapeutic target against tuberculosis. Sci Immunol 2.

Chouchani, E.T., Pell, V.R., Gaude, E., Aksentijević, D., Sundier, S.Y., Robb, E.L., Logan, A., Nadtochiy, S.M., Ord, E.N.J., Smith, A.C., et al. (2014). Ischaemic accumulation of succinate controls reperfusion injury through mitochondrial ROS. Nature 515, 431-435.

Clausen, B.E., Burkhardt, C., Reith, W., Renkawitz, R., and Förster, I. (1999). Conditional gene targeting in macrophages and granulocytes using LysMcre mice. Transgenic Res 8, 265-277.

Cohen, S.B., Gern, B.H., Delahaye, J.L., Adams, K.N., Plumlee, C.R., Winkler, J.K., Sherman, D.R., Gerner, M.Y., and Urdahl, K.B. (2018). Alveolar Macrophages Provide an Early Mycobacterium tuberculosis Niche and Initiate Dissemination. Cell Host Microbe 24, 439446.e434.

Cumming, B.M., Addicott, K.W., Adamson, J.H., and Steyn, A.J. (2018). Mycobacterium tuberculosis induces decelerated bioenergetic metabolism in human macrophages. Elife 7.

Daniel, J., Maamar, H., Deb, C., Sirakova, T.D., and Kolattukudy, P.E. (2011). Mycobacterium tuberculosis uses host triacylglycerol to accumulate lipid droplets and acquires a dormancy-like phenotype in lipid-loaded macrophages. PLoS Pathog 7, e1002093. 
908 Dézsi, C.A. (2016). Trimetazidine in Practice: Review of the Clinical and Experimental 909 Evidence. Am J Ther 23, e871-879.

910 Divakaruni, A.S., Hsieh, W.Y., Minarrieta, L., Duong, T.N., Kim, K.K.O., Desousa, B.R., 911 Andreyev, A.Y., Bowman, C.E., Caradonna, K., Dranka, B.P., et al. (2018). Etomoxir Inhibits 912 Macrophage Polarization by Disrupting CoA Homeostasis. Cell Metab 28, 490-503.e497.

913 Duman, C., Yaqubi, K., Hoffmann, A., Acikgöz, A.A., Korshunov, A., Bendszus, M., Herold914 Mende, C., Liu, H.K., and Alfonso, J. (2019). Acyl-CoA-Binding Protein Drives Glioblastoma 915 Tumorigenesis by Sustaining Fatty Acid Oxidation. Cell Metab 30, 274-289.e275.

916 Escoll, P., and Buchrieser, C. (2019). Metabolic reprogramming: an innate cellular defence 917 mechanism against intracellular bacteria? Curr Opin Immunol 60, 117-123.

918 Fernández-Agüera, M.C., Gao, L., González-Rodríguez, P., Pintado, C.O., Arias-Mayenco, I., 919 García-Flores, P., García-Pergañeda, A., Pascual, A., Ortega-Sáenz, P., and López-Barneo, J. 920 (2015). Oxygen Sensing by Arterial Chemoreceptors Depends on Mitochondrial Complex I 921 Signaling. Cell Metab 22, 825-837.

922 Galván-Peña, S., and O'Neill, L.A. (2014). Metabolic reprograming in macrophage polarization. 923 Front Immunol 5, 420.

924 Garaude, J., Acín-Pérez, R., Martínez-Cano, S., Enamorado, M., Ugolini, M., Nistal-Villán, E., 925 Hervás-Stubbs, S., Pelegrín, P., Sander, L.E., Enríquez, J.A., et al. (2016). Mitochondrial 926 respiratory-chain adaptations in macrophages contribute to antibacterial host defense. Nat 927 Immunol 17, 1037-1045.

928 Gleeson, L.E., Sheedy, F.J., Palsson-McDermott, E.M., Triglia, D., O'Leary, S.M., O'Sullivan, 929 M.P., O'Neill, L.A., and Keane, J. (2016). Cutting Edge: Mycobacterium tuberculosis Induces 930 Aerobic Glycolysis in Human Alveolar Macrophages That Is Required for Control of 931 Intracellular Bacillary Replication. J Immunol 196, 2444-2449.

932 Gonzalez-Hurtado, E., Lee, J., Choi, J., Selen Alpergin, E.S., Collins, S.L., Horton, M.R., and 933 Wolfgang, M.J. (2017). Loss of macrophage fatty acid oxidation does not potentiate systemic 934 metabolic dysfunction. Am J Physiol Endocrinol Metab 312, E381-E393.

935 Hall, C.J., Boyle, R.H., Astin, J.W., Flores, M.V., Oehlers, S.H., Sanderson, L.E., Ellett, F., 936 Lieschke, G.J., Crosier, K.E., and Crosier, P.S. (2013). Immunoresponsive gene 1 augments 937 bactericidal activity of macrophage-lineage cells by regulating $\beta$-oxidation-dependent 938 mitochondrial ROS production. Cell Metab 18, 265-278.

939 Harpey, C., Clauser, P., Labrid, C., Freyria, J.-L., and Poirier, J.-P. (1988). Trimetazidine, A 940 Cellular Anti-ischemic Agent. Cardiovascular Drug Reviews 6, 292-312.

941 Huang, L., Nazarova, E.V., Tan, S., Liu, Y., and Russell, D.G. (2018). Growth of 942 Mycobacterium tuberculosis in vivo segregates with host macrophage metabolism and ontogeny. 943 J Exp Med 215, 1135-1152. 
Jha, A.K., Huang, S.C., Sergushichev, A., Lampropoulou, V., Ivanova, Y., Loginicheva, E., Chmielewski, K., Stewart, K.M., Ashall, J., Everts, B., et al. (2015). Network integration of parallel metabolic and transcriptional data reveals metabolic modules that regulate macrophage polarization. Immunity 42, 419-430.

Kelly, B., and O'Neill, L.A. (2015). Metabolic reprogramming in macrophages and dendritic cells in innate immunity. Cell Res 25, 771-784.

950 Kim, Y.S., Lee, H.M., Kim, J.K., Yang, C.S., Kim, T.S., Jung, M., Jin, H.S., Kim, S., Jang, J., 951 Oh, G.T., et al. (2017). PPAR- $\alpha$ Activation Mediates Innate Host Defense through Induction of 952 TFEB and Lipid Catabolism. J Immunol 198, 3283-3295.

Köster, S., Upadhyay, S., Chandra, P., Papavinasasundaram, K., Yang, G., Hassan, A., Grigsby, S.J., Mittal, E., Park, H.S., Jones, V., et al. (2017). Mycobacterium tuberculosis is protected from NADPH oxidase and LC3-associated phagocytosis by the LCP protein CpsA. Proc Natl Acad Sci 956 U S A 114, E8711-E8720.

Lachmandas, E., Beigier-Bompadre, M., Cheng, S.C., Kumar, V., van Laarhoven, A., Wang, X., Ammerdorffer, A., Boutens, L., de Jong, D., Kanneganti, T.D., et al. (2016). Rewiring cellular metabolism via the AKT/mTOR pathway contributes to host defence against Mycobacterium

961 Lampropoulou, V., Sergushichev, A., Bambouskova, M., Nair, S., Vincent, E.E., Loginicheva, 962 E., Cervantes-Barragan, L., Ma, X., Huang, S.C., Griss, T., et al. (2016). Itaconate Links Inhibition of Succinate Dehydrogenase with Macrophage Metabolic Remodeling and Regulation of Inflammation. Cell Metab 24, 158-166. Jiang, H., MacArthur, M.R., et al. (2019). Glycerol phosphate shuttle enzyme GPD2 regulates macrophage inflammatory responses. Nat Immunol 20, 1186-1195. failure. Cardiovasc Res 90, 202-209.

972 Manzanillo, P.S., Ayres, J.S., Watson, R.O., Collins, A.C., Souza, G., Rae, C.S., Schneider, D.S., 973 Nakamura, K., Shiloh, M.U., and Cox, J.S. (2013). The ubiquitin ligase parkin mediates

974 resistance to intracellular pathogens. Nature 501, 512-516.

975 Manzanillo, P.S., Shiloh, M.U., Portnoy, D.A., and Cox, J.S. (2012). Mycobacterium 976 tuberculosis activates the DNA-dependent cytosolic surveillance pathway within macrophages. 977 Cell Host Microbe 11, 469-480.

978 Marrero, J., Rhee, K.Y., Schnappinger, D., Pethe, K., and Ehrt, S. (2010). Gluconeogenic carbon 979 flow of tricarboxylic acid cycle intermediates is critical for Mycobacterium tuberculosis to 980 establish and maintain infection. Proc Natl Acad Sci U S A 107, 9819-9824. 
981 Martin, C.J., Booty, M.G., Rosebrock, T.R., Nunes-Alves, C., Desjardins, D.M., Keren, I., 982 Fortune, S.M., Remold, H.G., and Behar, S.M. (2012). Efferocytosis is an innate antibacterial 983 mechanism. Cell Host Microbe 12, 289-300.

984 Martinez, J., Malireddi, R.K., Lu, Q., Cunha, L.D., Pelletier, S., Gingras, S., Orchard, R., Guan, 985 J.L., Tan, H., Peng, J., et al. (2015). Molecular characterization of LC3-associated phagocytosis 986 reveals distinct roles for Rubicon, NOX2 and autophagy proteins. Nat Cell Biol 17, 893-906.

987 Michelucci, A., Cordes, T., Ghelfi, J., Pailot, A., Reiling, N., Goldmann, O., Binz, T., Wegner, 988 A., Tallam, A., Rausell, A., et al. (2013). Immune-responsive gene 1 protein links metabolism to 989 immunity by catalyzing itaconic acid production. Proc Natl Acad Sci U S A 110, 7820-7825.

990 Mills, E.L., Kelly, B., Logan, A., Costa, A.S.H., Varma, M., Bryant, C.E., Tourlomousis, P., 991 Däbritz, J.H.M., Gottlieb, E., Latorre, I., et al. (2016). Succinate Dehydrogenase Supports 992 Metabolic Repurposing of Mitochondria to Drive Inflammatory Macrophages. Cell 167, 457993 470.e413.

994 Munoz-Elias, E.J., and McKinney, J.D. (2005). Mycobacterium tuberculosis isocitrate lyases 1 995 and 2 are jointly required for in vivo growth and virulence. Nat Med 11, 638-644.

996 Nair, S., Huynh, J.P., Lampropoulou, V., Loginicheva, E., Esaulova, E., Gounder, A.P., Boon, 997 A.C.M., Schwarzkopf, E.A., Bradstreet, T.R., Edelson, B.T., et al. (2018). Irgl expression in 998 myeloid cells prevents immunopathology during M. tuberculosis infection. J Exp Med 215, 999 1035-1045.

1000 Napier, R.J., Rafi, W., Cheruvu, M., Powell, K.R., Zaunbrecher, M.A., Bornmann, W., Salgame, 1001 P., Shinnick, T.M., and Kalman, D. (2011). Imatinib-sensitive tyrosine kinases regulate 1002 mycobacterial pathogenesis and represent therapeutic targets against tuberculosis. Cell Host 1003 Microbe 10, 475-485.

1004 Nazarewicz, R.R., Dikalova, A.E., Bikineyeva, A., and Dikalov, S.I. (2013). Nox2 as a potential 1005

1006

1007 Nunes, P., Demaurex, N., and Dinauer, M.C. (2013). Regulation of the NADPH oxidase and 1008 associated ion fluxes during phagocytosis. Traffic 14, 1118-1131.

1009 Olive, A.J., Smith, C.M., Kiritsy, M.C., and Sassetti, C.M. (2018). The Phagocyte Oxidase 1010 Controls Tolerance to Mycobacterium tuberculosis. J Immunol 201, 1705-1716.

1011 Ouimet, M., Koster, S., Sakowski, E., Ramkhelawon, B., van Solingen, C., Oldebeken, S., 1012 Karunakaran, D., Portal-Celhay, C., Sheedy, F.J., Ray, T.D., et al. (2016). Mycobacterium 1013 tuberculosis induces the miR-33 locus to reprogram autophagy and host lipid metabolism. Nat 1014 Immunol 17, 677-686.

1015 Pandey, A.K., and Sassetti, C.M. (2008). Mycobacterial persistence requires the utilization of 1016 host cholesterol. Proc Natl Acad Sci U S A 105, 4376-4380. 
1017 Parihar, S.P., Guler, R., Khutlang, R., Lang, D.M., Hurdayal, R., Mhlanga, M.M., Suzuki, H., 1018 Marais, A.D., and Brombacher, F. (2014). Statin therapy reduces the mycobacterium tuberculosis 1019 burden in human macrophages and in mice by enhancing autophagy and phagosome maturation. 1020 J Infect Dis 209, 754-763.

1021 Peyron, P., Vaubourgeix, J., Poquet, Y., Levillain, F., Botanch, C., Bardou, F., Daffe, M., Emile, 1022 1023 J.F., Marchou, B., Cardona, P.J., et al. (2008a). Foamy macrophages from tuberculous patients' granulomas constitute a nutrient-rich reservoir for M. tuberculosis persistence. PLoS Pathog 4, e1000204.

1025

1026

1027

1028

1029

1030

1031

1032

1033

1034

1035

1036

1037

1038

1039

1040

1041

1042

1043

1044

1045

1046

1047

1048

1049

1050

1051

1052

Peyron, P., Vaubourgeix, J., Poquet, Y., Levillain, F., Botanch, C., Bardou, F., Daffé, M., Emile, J.F., Marchou, B., Cardona, P.J., et al. (2008b). Foamy macrophages from tuberculous patients' granulomas constitute a nutrient-rich reservoir for M. tuberculosis persistence. PLoS Pathog 4, e1000204.

Pinegin, B., Vorobjeva, N., Pashenkov, M., and Chernyak, B. (2018). The role of mitochondrial ROS in antibacterial immunity. J Cell Physiol 233, 3745-3754.

Russell, D.G., Cardona, P.J., Kim, M.J., Allain, S., and Altare, F. (2009). Foamy macrophages and the progression of the human tuberculosis granuloma. Nat Immunol 10, 943-948.

Scialò, F., Fernández-Ayala, D.J., and Sanz, A. (2017). Role of Mitochondrial Reverse Electron Transport in ROS Signaling: Potential Roles in Health and Disease. Front Physiol 8, 428.

Scialò, F., Sriram, A., Fernández-Ayala, D., Gubina, N., Lõhmus, M., Nelson, G., Logan, A., Cooper, H.M., Navas, P., Enríquez, J.A., et al. (2016). Mitochondrial ROS Produced via Reverse Electron Transport Extend Animal Lifespan. Cell Metab 23, 725-734.

Shi, L., Salamon, H., Eugenin, E.A., Pine, R., Cooper, A., and Gennaro, M.L. (2015). Infection with Mycobacterium tuberculosis induces the Warburg effect in mouse lungs. Sci Rep 5, 18176.

Singh, V., Jamwal, S., Jain, R., Verma, P., Gokhale, R., and Rao, K.V. (2012). Mycobacterium tuberculosis-driven targeted recalibration of macrophage lipid homeostasis promotes the foamy phenotype. Cell Host Microbe 12, 669-681.

Singhal, A., Jie, L., Kumar, P., Hong, G.S., Leow, M.K., Paleja, B., Tsenova, L., Kurepina, N., Chen, J., Zolezzi, F., et al. (2014). Metformin as adjunct antituberculosis therapy. Sci Transl Med 6, 263 ra159.

Sun, J., Singh, V., Lau, A., Stokes, R.W., Obregón-Henao, A., Orme, I.M., Wong, D., Av-Gay, Y., and Hmama, Z. (2013). Mycobacterium tuberculosis nucleoside diphosphate kinase inactivates small GTPases leading to evasion of innate immunity. PLoS Pathog 9, e1003499.

Upadhyay, S., and Philips, J.A. (2019). LC3-associated phagocytosis: host defense and microbial response. Curr Opin Immunol 60, 81-90.

Van den Bossche, J., O'Neill, L.A., and Menon, D. (2017). Macrophage Immunometabolism: Where Are We (Going)? Trends Immunol 38, 395-406. 
1053 Vorobjeva, N., Prikhodko, A., Galkin, I., Pletjushkina, O., Zinovkin, R., Sud'ina, G., Chernyak, 1054 B., and Pinegin, B. (2017). Mitochondrial reactive oxygen species are involved in

1055 chemoattractant-induced oxidative burst and degranulation of human neutrophils in vitro. Eur J

1056 Cell Biol 96, 254-265.

1057 Wang, T., Fahrmann, J.F., Lee, H., Li, Y.J., Tripathi, S.C., Yue, C., Zhang, C., Lifshitz, V., 1058 Song, J., Yuan, Y., et al. (2018). JAK/STAT3-Regulated Fatty Acid $\beta$-Oxidation Is Critical for 1059 Breast Cancer Stem Cell Self-Renewal and Chemoresistance. Cell Metab 27, 1357.

1060 Wang, Y., Palmfeldt, J., Gregersen, N., Makhov, A.M., Conway, J.F., Wang, M., McCalley, 1061 S.P., Basu, S., Alharbi, H., St Croix, C., et al. (2019). Mitochondrial fatty acid oxidation and the 1062 electron transport chain comprise a multifunctional mitochondrial protein complex. J Biol Chem $1063294,12380-12391$.

1064 West, A.P., Brodsky, I.E., Rahner, C., Woo, D.K., Erdjument-Bromage, H., Tempst, P., Walsh, 1065 M.C., Choi, Y., Shadel, G.S., and Ghosh, S. (2011). TLR signalling augments macrophage 1066 bactericidal activity through mitochondrial ROS. Nature 472, 476-480.

1067 Wolf, A.J., Linas, B., Trevejo-Nuñez, G.J., Kincaid, E., Tamura, T., Takatsu, K., and Ernst, J.D. 1068 (2007). Mycobacterium tuberculosis infects dendritic cells with high frequency and impairs their 1069 function in vivo. J Immunol 179, 2509-2519.

1070 Wong, K.W., and Jacobs, W.R. (2011). Critical role for NLRP3 in necrotic death triggered by 1071 Mycobacterium tuberculosis. Cell Microbiol 13, 1371-1384.

1072 Xue, J., Schmidt, S.V., Sander, J., Draffehn, A., Krebs, W., Quester, I., De Nardo, D., Gohel, 1073 T.D., Emde, M., Schmidleithner, L., et al. (2014). Transcriptome-based network analysis reveals 1074 a spectrum model of human macrophage activation. Immunity 40, 274-288. 